\title{
Stellar impact on disequilibrium chemistry and observed spectra of hot Jupiter atmospheres
}

\author{
D. Shulyak ${ }^{1}$, L. M. Lara ${ }^{2}$, M. Rengel ${ }^{1}$, and N.-E. Nèmec ${ }^{1}$ \\ ${ }^{1}$ Max-Planck Institut für Sonnensystemforschung, Justus-von-Liebig-Weg 3, 37077, Göttingen, Germany \\ e-mail: shulyak@mps.mpg.de \\ 2 Instituto de Astrofísica de Andalucía - CSIC, c/ Glorieta de la Astronomía s/n, 18008 Granada, Spain
}

Received 29 November 2019 / Accepted 30 April 2020

\begin{abstract}
Aims. We study the effect of disequilibrium processes (photochemistry and vertical transport) on mixing ratio profiles of neutral species and on the simulated spectra of a hot Jupiter exoplanet that orbits stars of various spectral types. We additionally address the impact of stellar activity that should be present, to various degrees, in all stars with convective envelopes.

Methods. We used the VULCAN chemical kinetic code to compute number densities of species in irradiated planetary atmospheres. The temperature-pressure profile of the atmosphere was computed with the HELIOS code. We also utilized the $\tau$-REx forward model to predict the spectra of planets in primary and secondary eclipses. In order to account for the stellar activity, we made use of the observed solar extreme ultraviolet (XUV) spectrum taken from Virtual Planetary Laboratory as a proxy for an active sun-like star. Results. We find large changes in the mixing ratios of most chemical species in planets orbiting A-type stars, which radiate strong XUV flux thereby inducing a very effective photodissociation. For some species, these changes can propagate very deep into the planetary atmosphere to pressures of around 1 bar. To observe disequilibrium chemistry we favor hot Jupiters with temperatures $T_{\mathrm{eq}}=1000 \mathrm{~K}$ and ultra-hot Jupiters, with $T_{\mathrm{eq}} \approx 3000 \mathrm{~K}$, which also have temperature inversion in their atmospheres. On the other hand, disequilibrium calculations predict no noticeable changes in spectra of planets with intermediate temperatures. We also show that stellar activity similar to that of the modern Sun drives important changes in mixing ratio profiles of atmospheric species. However, these changes take place at very high atmospheric altitudes and thus do not affect predicted spectra. Finally, we estimate that the effect of disequilibrium chemistry in planets orbiting nearby bright stars could be robustly detected and studied with future missions with spectroscopic capabilities in infrared such as James Webb Space Telescope and ARIEL.
\end{abstract}

Key words. planets and satellites: atmospheres - planets and satellites: composition - stars: activity - methods: numerical

\section{Introduction}

Since the moment of their birth and the accretion of first atmospheres, the evolution of planets is closely related to the evolution of their host stars through a wealth of different processes that are usually summarized under a single definition of the star-planet interaction. For example, planet evolution includes the tidal interaction between the star and the planet (Debes \& Jackson 2010) with two planets detected close to their tidal disruption limit (Delrez et al. 2016; Birkby et al. 2014). If the planet is very close to its host star, the interaction between the stellar and planetary magnetic fields can induce active regions on the surface of the host (Cauley et al. 2019). In rocky planets, strong gravitational tides and/or induction heating can significantly enhance volcanic activity and hence outgassing, which can create dense Venus-like atmospheres (Kislyakova et al. 2017). Finally, stellar radiation shapes the temperature distribution in planetary atmospheres; stellar activity produces strong nonthermal radiation, such as X-ray and ultraviolet (UV) to extreme ultraviolet (XUV), and energetic particle flux (coronal mass ejections; CME), which all drive atmospheric chemistry out of equilibrium and eventually control processes of atmospheric erosion (Johnstone et al. 2019; Dwivedi et al. 2019). Among all these processes, stellar radiation is of particular interest because it acts on the atmosphere of every planet and its impact on, for example, atmospheric composition could directly be studied by analyzing the spectra of the planetary atmosphere.

Giant planets at short orbital distances, such as hot Jupiters (HJs) and smaller Neptune-size planets, receive strong radiation from their host stars, which raises atmospheric temperatures to a thousand and, in some extreme cases of so-called ultra-hot Jupiters (UHJ), to even $T_{\text {eq }}=4000 \mathrm{~K}$ for the famous KELT-9b planet (Yan \& Henning 2018). This leads to an intense photodissociation of molecules at high altitudes in their atmospheres, such that the expected concentrations of molecular and atomic species may start to strongly deviate from their equilibrium values. This deviation becomes highly noticeable when, additionally to photochemistry, the transport processes (e.g., molecular diffusion) are taken into account. Hot Jupiters are the beststudied class of exoplanets to date because they are easy to observe thanks to their short orbital periods, large sizes, and hot atmospheric temperatures; the hot temperatures make the planetto-star flux contrast relatively large. This makes HJs the most promising targets to study atmospheric chemistry in detail with current and future instruments and missions.

Two main disequilibrium processes modify abundances within exoplanetary atmospheres. The first is photochemistry, which includes dissociation and ionization of atmospheric constitutes by stellar XUV radiation. The second is vertical mixing, which regulates how the atmospheric species segregate within the atmosphere under the action of competing processes of 
molecular diffusion and turbulent mixing. The consequences of disequilibrium chemistry on the atmospheric composition of different kinds of exoplanets have been explored by numerous groups in the past. Early studies specifically addressed questions of photochemically driven production of atomic species and thermospheric escape in HJ atmospheres (Liang et al. 2003; Yelle 2004; Koskinen et al. 2007; García Muñoz 2007) as well as pathway analyses of different photochemical products (e.g., Zahnle et al. 2009; Line et al. 2010). These studies led to the development of new models with improved photochemical and kinetic calculations. These models were used to study disequilibrium processes in objects of different masses and temperatures: in terrestrial exoplanet atmospheres using benchmark cases of Mars and Earth (e.g., Hu et al. 2012), super-Earths (GJ 1214 b, Miller-Ricci Kempton et al. 2012), hot Neptunes (GJ 436 b, Line et al. 2011), and HJs (Miguel \& Kaltenegger 2014; Kopparapu et al. 2012). In-depth analysis of disequilibrium effects, including realistic treatment of activity driven stellar XUV radiation, and validation of chemical networks was performed for the two benchmark cases of HD 209458 b and HD 189733 b. Both planets have close equilibrium temperatures $\left(T_{\mathrm{eq}}=1200 \mathrm{~K}\right.$ and $T_{\text {eq }}=1500 \mathrm{~K}$, respectively), but have orbiting stars of different types and ages (Moses et al. 2011; Venot et al. 2012). In UHJs, such as KELT-9, high temperatures and strong irradiation causes large amounts of atoms and ions to be present in upper atmospheric layers, as was predicted by Kitzmann et al. (2018) and later detected using high-resolution spectroscopic observations by Hoeijmakers et al. (2019). However, these two studies did not attempt detailed predictions of the impact of disequilibrium processes on the spectra of KELT-9. Finally, a possibility to study disequilibrium chemistry in atmospheres of three planets (HD 189733 b, WASP-80 b, and GJ 436 b, all having $T_{\text {eq }}<1200 \mathrm{~K}$ ) with future space missions was carried out in Blumenthal et al. (2018), concluding that, for example, with the James Webb Space Telescope (JWST) it will be possible to robustly constrain the difference between equilibrium and disequilibrium chemistry. Analyzing chemistry in HJs provides an important test for existing kinetic models as they include many poorly known parameters (e.g., turbulent diffusion coefficients, photodissociation cross-section, reaction rate coefficients, and choice of the kinetic network.). When the atmosphere of a planet is driven away from its chemical equilibrium, the changes in molecular concentrations start to affect the temperature structure of the atmosphere via the changes in local opacity. That is, the initially assumed temperature structure may change if the changes in opacity are strong enough. Ideally, this nonlinear response of the atmospheric structure to the disequilibrium processes must be taken into account in a self-consistent way by solving for the temperature structure and number densities simultaneously. This complicates the problem enormously because solving for the temperature structure requires knowledge of frequency integrated properties of the radiation field at every point in the planetary atmosphere. As a result, many works addressed only the effect of photochemistry and kinetics on the changes in mixing ratios and observed characteristic of some benchmark HJs (see, e.g., Hobbs et al. 2019; Venot et al. 2012; Moses et al. 2011). Recently, Molaverdikhani et al. (2019a) investigated the effect of disequilibrium chemistry on the observed properties of planetary atmospheres using an extensive grid of models that covered a very large range of planetary and stellar parameters. However, they did not specifically address the effects of stellar activity as a function of stellar age (limiting only to the XUV flux of the present Sun) and of inverted temperature profiles expected for UHJs.
Self-consistent kinetic-structure models of planetary atmospheres remain a challenging task, but the first models have already been successfully developed in the past. For instance, Drummond et al. (2016) used the ATMO code that can simultaneously solve for kinetic and radiative-convective equilibrium to study the temperature structure and emission spectra of HJs. These authors conclude that not accounting for the radiativeconvective equilibrium can lead to an overestimation of the effect of disequilibrium chemistry on the observed spectra. The authors highlight the importance of self-consistent models because, although the emission spectra did not show large changes between equilibrium and disequilibrium calculations, the mixing ratios and atmospheric temperature were both highly affected by photodissociation and molecular diffusion processes.

In this work we aim at a systematic analysis of disequilibrium chemistry in an atmosphere of a Jupiter size exoplanet that orbits stars of different spectral types. We do not attempt to compute self-consistent kinetic models similar to Drummond et al. (2016). Instead, we take a first step toward extending our study of disequilibrium chemistry considering various stellar spectral types and we make predictions for both emission and transmission observations. Moreover, studying the impact of disequilibrium chemistry, we additionally investigate the impact of stellar activity on atmospheres of HJs orbiting young sun-like stars that maintain a high level of XUV radiation during early stages of their evolution.

\section{Methods}

\subsection{Atmospheric pressure-temperature profiles}

We utilized the HELIOS ${ }^{1}$ code to compute temperature structure of atmospheres using self-consistent radiative-convective iteration (Malik et al. 2017). The typical inputs for the HELIOS are the parameters of the planet (mass, radius, atmospheric abundances, and semimajor axis) and its host star (radius and effective temperature) and we used the blackbody approximation for the stellar spectrum. Our calculation setup included 100 atmospheric layers distributed logarithmically between $10^{-9}$ bar and 100 bar and we assumed global redistribution of the heat across day and night sides of the planet.

Modeling atmospheres of UHJs deserves additional attention. Intense XUV radiation of hosts stars drives very efficient photodissociation of molecules, which then enhances concentrations of atomic species and their ions in the atmospheres of these planets (Casasayas-Barris et al. 2019). The amount of these metals could be so large that they begin to play a dominant role in shaping the P-T structure by further absorbing XUV stellar flux. Most of the absorbed energy goes into the kinetic energy of the ionized atoms and electrons. The result of this process is the sudden temperature raise detected in corresponding atmospheric layers (Malik et al. 2019; Arcangeli et al. 2018). It was then understood that a proper modeling of UHJ atmospheres requires inclusion of opacity due to metals and their ions. Unfortunately, in its current stage the opacity tables included in the public version of the HELIOS code do not include continuum and line opacity due to atoms. We therefore considered two approaches. First, because our analysis has an exploratory nature and does not involve direct comparisons with real data, we still used HELIOS to compute T-P profiles for most irradiated planets. Second, we considered analytical T-P profiles after Parmentier \& Guillot (2014) to explore the effect of temperature inversion

1 https://github.com/exoclime/HELIOS 


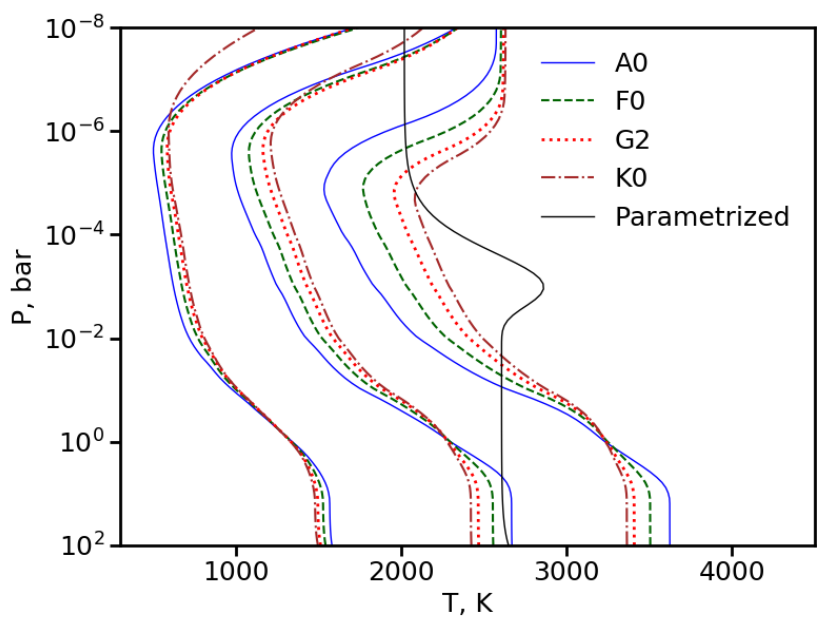

Fig. 1. Temperature profiles of a Jupiter size planet around three types of host stars ( $\mathrm{A} 0$ - solid blue line, $\mathrm{F} 0$ - dashed green line, $\mathrm{G} 2$ - dotted red line, and K0 - dash-dotted brown line) calculated with HELIOS and grouped according to the equilibrium temperatures of $1000 \mathrm{~K}$ (left), $2000 \mathrm{~K}$ (middle), and $3000 \mathrm{~K}$ (right), respectively. The parameterized profile after Parmentier \& Guillot (2014) with temperature inversion in upper atmospheric layers is shown with a full black line.

on the chemical profiles and predicted spectra of UHJ. Since we are interested in studying general changes in predicted spectra of planets with temperature inversion in their atmospheres, we cose a parameterized T-P model with an inversion at the millibar level. We note that we do not compare the resulting synthetic spectra obtained with the temperature profiles from HELIOS with those from parameterized models. The temperature inversion can be obtained either with Parmentier \& Guillot (2014) or, for example, with another commonly used parameterization after Guillot (2010). An extensive use of these parameterized models can be found in, for example, Parmentier et al. (2015) and Venot et al. (2015).

Our Fig. 1 illustrates examples of temperature profiles corresponding to $T_{\text {eq }}=1000 \mathrm{~K}, 2000 \mathrm{~K}, 3000 \mathrm{~K}$, and inverted $T_{\text {eq }}=3000 \mathrm{~K}$, respectively, for a Jupiter size planet orbiting stars of different spectral types. We note the difference between temperature profiles introduced by stellar types. This difference is obviously absent in the case of the parameterized profile. The equilibrium temperatures were estimated assuming planetary albedo $\alpha=0.03$ (Sudarsky et al. 2000).

\subsection{Chemical kinetic model}

The vertical distribution of neutral atmospheric species in the atmosphere is obtained by solving the coupled kinetic equations for species containing $\mathrm{C}, \mathrm{H}, \mathrm{O}$, and $\mathrm{N}$ using the VULCAN code ${ }^{2}$ (Tsai et al. 2017). All absorption and photodissociation cross sections are from the Leiden Observatory database ${ }^{3}$ (see also Heays et al. 2017), except $\mathrm{NO}_{3}, \mathrm{HNO}_{3}, \mathrm{HNO}_{2}, \mathrm{~N}_{2} \mathrm{O}$, and $\mathrm{NO}_{2}$; these are from Huebner \& Mukherjee (2015), Huebner et al. (1992), and Huebner \& Carpenter (1979), where $\mathrm{NO}_{2}$ combines $\mathrm{NO} / \mathrm{O}\left({ }^{1} \mathrm{D}\right)$ and $\mathrm{NO} / \mathrm{O}$ to obtain the total absorption cross section. $\mathrm{N}_{2} \mathrm{H}_{4}$ is from MPI-Mainz UV/VIS Spectral Atlas ${ }^{4}$. We assumed that the metallicity of the exoplanetary atmosphere is solar (Lodders et al. 2009), that is $\mathrm{C} / \mathrm{H}=2.45 \times 10^{-4}, \mathrm{O} / \mathrm{H}=4.57 \times 10^{-4}$, and $\mathrm{N} / \mathrm{H}=6.03 \times 10^{-5}$. This renders a C-to-O ratio to 0.54 . The

2 https://github.com/exoclime/VULCAN

3 http://home.strw. leidenuniv.nl/ ewine/photo

4 http://satellite.mpic.de/spectral_atlas/index.html

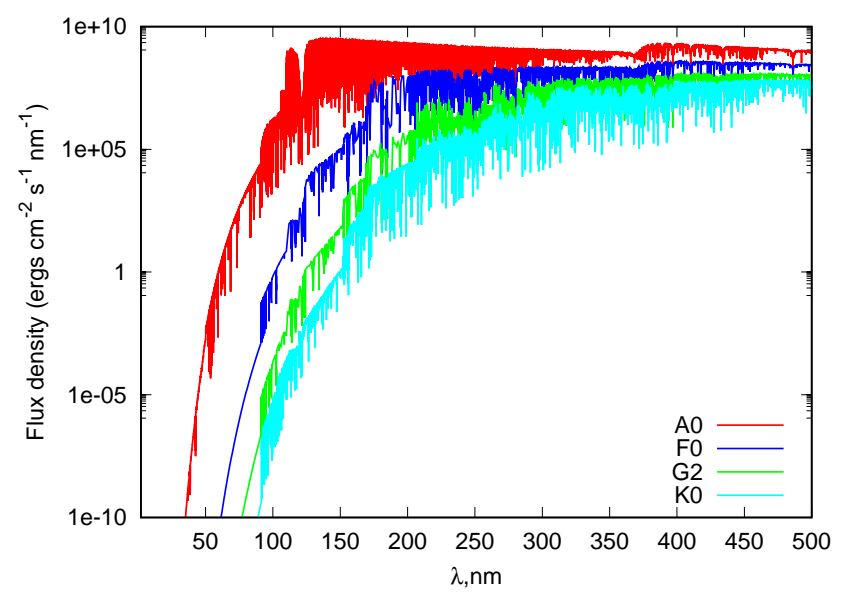

Fig. 2. Flux density at the stellar surface for $\mathrm{A} 0, \mathrm{~F} 0, \mathrm{G} 2$, and $\mathrm{K} 0$ stars as obtained from PHOENIX model library (Husser et al. 2013).

chemical kinetic equations are solved between 100 and $10^{-8}$ bar with boundary conditions of zero flux at both atmospheric levels. The thermal profile $\mathrm{T}(\mathrm{p})$ and the metallicity are the only inputs needed by FASTCHEM ${ }^{5}$ (Stock et al. 2018) to obtain the thermochemical equilibrium (EQ) abundances of the considered species. The obtained vertical profiles are then subject to photodissociation caused by the stellar flux, turbulent transport, and molecular diffusion with the VULCAN code

The stellar flux was taken from the PHOENIX library (Husser et al. 2013) and scaled according to the orbital distance of the planet and the size of the host star. Each stellar type that we considered in this study (A0, F0, G2, K0) was assumed to have solar metallicity. Figure 2 shows the spectral flux energy at the stellar surface at $\lambda \leqslant 500 \mathrm{~nm}$ as this is the spectral region essential for the bulk of the photodissociation processes.

We purposely ignored $\mathrm{M}$ dwarfs in our study because these stars are not expected to form many HJs (Obermeier et al. 2016; Mordasini et al. 2012). Up to now only a few Jupiter size planets were discovered around $\mathrm{M}$ dwarfs (Bayliss et al. 2018; Bakos et al. 2018; Hartman et al. 2015; Johnson et al. 2012). We note that all of these planet have $T_{\text {eq }}<1000 \mathrm{~K}$, which is outside of the temperature range considered in this work.

The turbulent transport is usually parameterized in 1D models by so-called eddy diffusion coefficient $K_{z z}$, which is associated with vertical mixing. As in Kitzmann et al. (2018), we assumed $K_{z z}=0.1 H_{\mathrm{p}} v_{z}$, where $H_{\mathrm{p}}$ refers to the atmospheric scale height and $v_{z}$ to the vertical velocity, which is approximated to be the atmospheric speed of sound $c_{\mathrm{s}}$. This consideration turns into $K_{z z}$ ranging from $4.3 \times 10^{9}$ to $2.4 \times 10^{10} \mathrm{~cm}^{2} \mathrm{~s}^{-1}$ for $T_{\text {eq }}$ between 1000 and $3000 \mathrm{~K}$. The molecular diffusion coefficients were computed following Moses et al. (2011, 2000) and these depend on temperature, total number density, and mass of the diffusing species. Other approaches can be taken as well (e.g., Chapman and Enskog equation from Poling et al. 2000).

\subsection{Predicting emission and transmission spectra of HJs}

In order to predict emission and transmission spectra of HJs we utilized the $\tau$-REx (Tau Retrieval for Exoplanets) software package (Waldmann et al. 2015b,a). This package uses up-todate molecular cross sections based on line lists provided by EXOMOL ${ }^{6}$ project (Tennyson \& Yurchenko 2012) and HITEMP

\footnotetext{
5 https://github.com/exoclime/FastChem

6 wWW . exomol. com
} 
(Rothman et al. 2010). We additionally used the HCN line list after Harris et al. (2006) provided by the EXOCLIME project ${ }^{7}$ and generated with the HELIOS-K code (Grimm \& Heng 2015). These cross sections are precomputed on a grid of temperatures and pressures and are stored in binary opacity tables that are available for a number of spectral resolutions. The continuum opacity includes Rayleigh scattering on molecules and collisionally-induced absorption due to $\mathrm{H}_{2}-\mathrm{H}_{2}$ and $\mathrm{H}_{2}-\mathrm{He}$ either after Abel et al. (2011, 2012) or Borysow et al. (2001); Borysow (2002); Borysow \& Frommhold (1989), respectively. We extended the public version of $\tau$ - $\mathrm{REx}^{8}$ by incorporating additional opacity sources essential for the atmospheres of UHJs. In particular, bound-free and free-free transitions of $\mathrm{H}^{-}$become one of the major continuum opacity contributors for the temperatures hotter than about $2000 \mathrm{~K}$. The cross sections of $\mathrm{H}^{-}$are from John (1988). We also included opacity due to free-free transitions of $\mathrm{He}^{-}$as well as Rayleigh scattering on $\mathrm{HI}$ atoms and Thomson scattering on free electrons. The $\mathrm{He}^{-}$cross sections are those originally from John (1968) using the polynomial fit by Carbon et al. (1969). Rayleigh scattering on $\mathrm{H} \mathrm{I}$ is calculated after Dalgarno (1962). All relevant numerical routines were extracted from the LLMODELS stellar model atmosphere code (Shulyak et al. 2004). At the temperatures of $\mathrm{HJs}_{\mathrm{s}}$ the $\mathrm{H}^{-}$is the dominant continuum opacity source that impacts the observed spectra of these planets (Arcangeli et al. 2018). However, at millibar pressures the $\mathrm{He}^{-}$opacity can become comparable or even stronger than that of $\mathrm{H}^{-}$for wavelengths longer than $1.6 \mu \mathrm{m}$, as shown in Fig. 3 (third panel from the bottom), where we display examples of continuum opacity coefficient at different altitudes in the atmosphere of a HJ with $T_{\text {eq }}=3000 \mathrm{~K}$. At even smaller pressures, electron scattering and Rayleigh scattering on $\mathrm{H}$ I atoms also become important contributors to the continuum opacity at particular wavelengths (top panel in Fig. 3). However, the contribution of $\mathrm{HI}$ and $\mathrm{e}^{-}$on the transmission spectra is marginal because their opacity is only strong at low pressures that are hardly probed by transmission spectroscopy. Thus, among all continuum opacity sources, only $\mathrm{H}^{-}$and $\mathrm{He}^{-}$significantly contribute to the predicted amplitude of the transmission spectra, as shown in the bottom plot of Fig. 3. When both continuum and line opacity are included, the effect of $\mathrm{He}^{-}$on predicted spectra is diluted by a much stronger opacity in molecular lines while $\mathrm{H}^{-}$contribution is still significant. Nevertheless, as can be seen from the second plot (from bottom) in Fig. 3, in optically thick layers the $\mathrm{He}^{-}$opacity could still be stronger than, for example, collision-induced absorption due to $\mathrm{H}_{2}-\mathrm{H}_{2}$ and $\mathrm{H}_{2}$ $\mathrm{He}$. We thus conclude that accurate calculation of atmospheric opacity requires $\mathrm{He}^{-}, \mathrm{HI}$, and $\mathrm{e}^{-}$opacity included especially at low pressures, following modern stellar atmosphere codes. However, observed transmission and emission spectra of HJs are hardly affected by these three opacity sources. Finally, we updated HELIOS with $\mathrm{He}^{-}$and $\mathrm{e}^{-}$opacity and found out that this has little impact on the atmospheric temperature structure (with a modification in local temperature of at most $\Delta T \approx 10 \mathrm{~K}$ ) and thus can be ignored; the original version of HELIOS already includes H I Rayleigh scattering.

\subsection{Stellar activity}

Dynamos in stars with outer convective envelopes can generate strong magnetic fields that interact with stellar convection and eventually dissipate their energy in magnetic reconnection

\footnotetext{
7 https://dev.opacity.iterativ.ch/\#/

8 https://github.com/ucl-exoplanets/TauREx_public
}
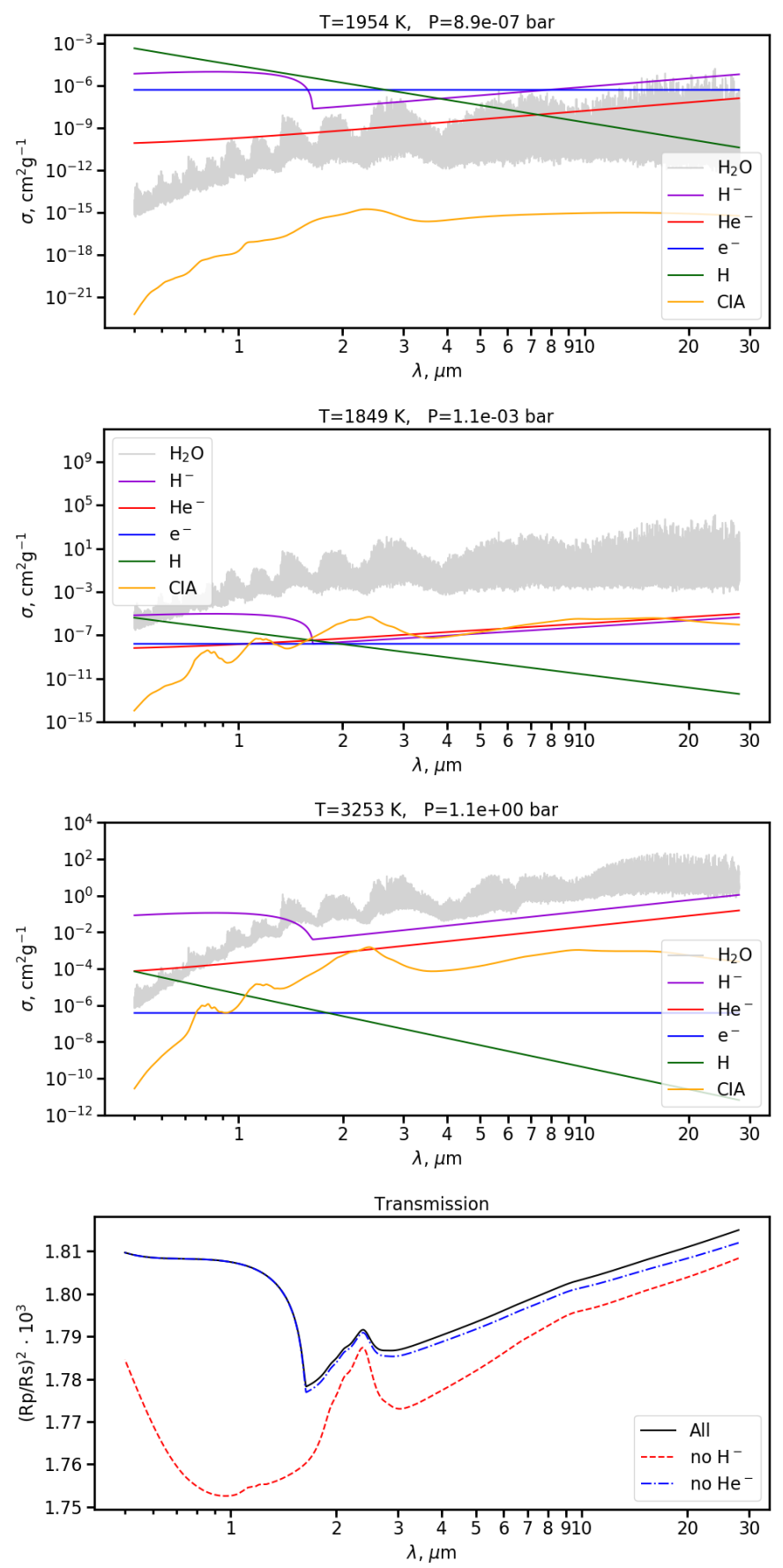

Fig. 3. Continuum opacity calculated for a $\mathrm{HJ}$ with $T_{\mathrm{eq}}=3000 \mathrm{~K}$ at three different altitude levels (with pressure decreasing from bottom to top). The opacity due to $\mathrm{H}_{2} \mathrm{O}$ is shown for comparison purposes. The bottom plot shows the transmission amplitude calculated including only continuum opacity sources (i.e., without line opacity) and illustrates the relative contribution of $\mathrm{H}^{-}$and $\mathrm{He}^{-}$absorption.

events. The latter heat up the regions above stellar photospheres and create chromospheres and coronae. High temperature in those atmospheric regions enhances stellar XUV flux.

During their life on the main sequence, stars with convective envelopes undergo two major regimes of their activity evolution. First, when stars reach the main sequence, they generally rotate very fast and their nonthermal emission is maximal. This is the regime of activity saturation because the amount of XUV flux does not change with time as the rotation rate keeps decreasing 
owing to magnetic braking. The level of magnetic activity in this regime is determined, to a very good approximation, by the thickness of the convective zone, which sets the amount of magnetic energy the star can generate (Christensen et al. 2009). As stars evolve they keep spinning down and after some time reach a critical rotation period above which the activity starts to desaturate and begins to decline as rotation rate of stars decreases further (non-saturated regime, see, e.g., Reiners et al. 2014; Pizzolato et al. 2003; Noyes et al. 1984). For instance, the Sun was more active in the past and its XUV flux was stronger, which potentially drove an enhanced erosion of atmospheres of the Earth and other planets during first several hundred million years after the formation of the solar system (Johnstone et al. 2019; Tu et al. 2015; Ribas et al. 2005; Güdel et al. 1997). Even now, at its relatively quiet state, the Sun produces enhanced XUV emission, which cannot be predicted by standard photospheric models.

Because most molecular photodissiociation cross sections are found in the short wavelength domain, it becomes evident that accurate knowledge of XUV radiation is essential when studying the atmospheric chemistry of planets orbiting stars with convective envelopes. Therefore, we extended our investigation of the $\mathrm{G} 2$ case by additionally accounting for the stellar activity. We note that some semi-empirical models of stellar chromospheres have been constructed in the past to mimic the amount of nonthermal radiation from convective stars (e.g., Fontenla et al. 2016; Vernazza et al. 1981). However, calculation of the outgoing radiation from these models requires complicated approaches including nonequilibrium effects on atoms and ions. Instead, we specifically chose the case of G2 star in our stellar sample because it resembles the temperature and radii properties of the Sun (Segura et al. 2003). Thus we used the observed solar radiation to address the impact of stellar activity on the atmospheric spectra of our test planet that orbits G2 star using Sun as a proxy of XUV radiation.

As a next step we studied the effect of an increased stellar activity for the G2 star when it was 100 Myr young. We used estimates by Claire et al. (2012) who investigated the evolution of the solar flux in time following Ribas et al. (2005). The corresponding routines were taken from the Virtual Planetary Laboratory web page ${ }^{9}$. According to the suggested scaling relations, a 100 Myr young Sun would have had high-energy emissions about 100 times larger than those of the modern Sun, whereas it was less luminous and smaller $\left(T_{\mathrm{eff}}(t=4.5 \mathrm{Gyr})=5778 \mathrm{~K}\right.$; $\left.T_{\text {eff }}(t=0.1 \mathrm{Gyr})=5650 \mathrm{~K}, R(t=0.1 \mathrm{Gyr})=0.876 R(t=4.5 \mathrm{Gyr})\right)$. The adopted radiation of the young and modern Sun at 1 AU are shown in Fig. 4.

\section{Results}

In the simulations that we present in this sect ion we consider a Jupiter size planet with three different equilibrium temperatures $T_{\text {eq }}$ of 1000,2000 , and $3000 \mathrm{~K}$, respectively. These temperatures correspond to typical conditions found in HJs $\left(T_{\mathrm{eq}} \leqslant 2000 \mathrm{~K}\right)$ and UHJs $\left(T_{\text {eq }}>2000 \mathrm{~K}\right)$; we note that the classification of UHJ in terms of their temperatures is a matter of debate. Each of these $T_{\text {eq }}$ can be reached at different distances from the parent star, depending on the spectral type of the later. We chose four types of stars: A0 $\left(T_{\text {eff }}=10800 \mathrm{~K}, R=2.5 R_{\odot}\right)$, F0 $\left(T_{\mathrm{eff}}=7200 \mathrm{~K}, R=1.3 R_{\odot}\right), \mathrm{G} 2\left(T_{\mathrm{eff}}=5800 \mathrm{~K}, R=1.0 R_{\odot}\right)$, and $\mathrm{K} 0\left(T_{\text {eff }}=5200 \mathrm{~K}, R=0.85 R_{\odot}\right)$ For the UHJ atmospheres, that is, for planets with $T_{\text {eq }}=3000 \mathrm{~K}$, we additionally considered

9 http://depts.washington.edu/naivpl/content/models/ solarflux

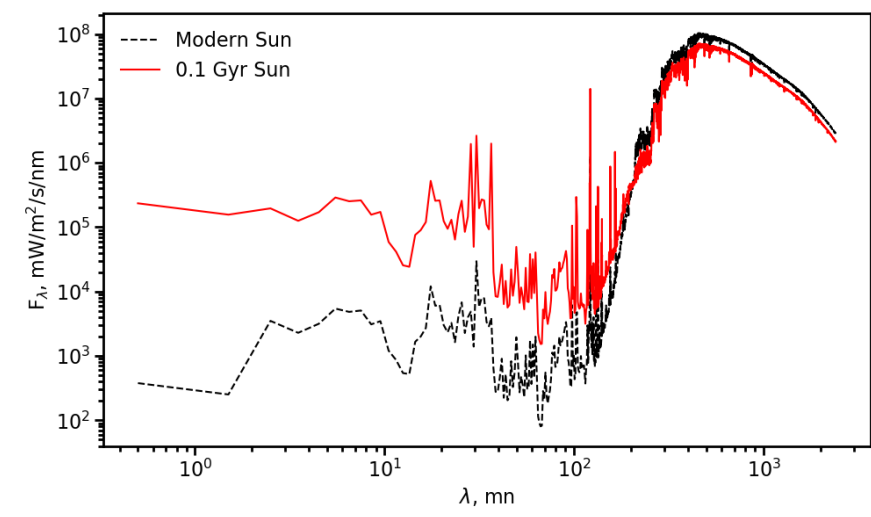

Fig. 4. Energy distribution of the modern (dashed line) and $0.1 \mathrm{Gyr}$ young Sun (solid line) at the top of the Earth atmosphere according to Claire et al. (2012).

a parameterized temperature profile with a temperature inversion at high altitudes. Throughout the paper we call models that corresponds to four different temperatures as T1, T2, T3, and T3-inverted, respectively (see Fig. 1). Below we investigate the impact of stellar types on atmospheric chemistry and spectra as predicted by EQ and photokinetic disequilibrium (DQ) models.

\subsection{Atmospheric chemistry}

We first look at the influence of stellar spectral types on the chemical composition. The corresponding plots are shown in the right panels of Figs. 5-8. First, we find that, regardless of the star type, for the coolest planet considered in this work (i.e., the $\mathrm{T} 1$ case), disequilibrium processes can affect the mixing ratio of molecules $\mathrm{NH}_{3}, \mathrm{CH}_{4}$, and $\mathrm{HCN}$ throughout the whole atmosphere and even down to optically thick layers that lay below 1 bar level. Also Moses et al. (2011) noted that for HJs HD 209458b and HD 189733b (planets orbiting main-sequence G0 and $\mathrm{K} 2$ stars, respectively, with $T_{\mathrm{eq}} \sim 1200 \mathrm{~K}$ ), molecules as $\mathrm{CO}_{2}, \mathrm{CO}, \mathrm{H}_{2} \mathrm{O}$, and $\mathrm{N}_{2}$ are relatively unaffected by disequilibrium chemistry. Our study extends this conclusion to hotter stars of spectral types $\mathrm{A} 0$ and $\mathrm{F} 2$.

When the planet is placed closer to the host star (i.e., cases $\mathrm{T} 2$ and T3), the higher XUV radiation by the A0 star compared to other stars considered in this model (see Fig. 2) induces a more efficient molecular photodissociation. This also produces a strong decrease of the species mixing ratios at low pressure levels where the radiation can penetrate. The characteristic sudden drop in molecular mixing ratios seen at around $P \approx 10^{-3}$ bar for the T3-inverted case (top right plots in Figs. 5-8) is caused by the corresponding temperature inversion that is present in the parameterized temperature profile (see Fig. 1). In this case, the mixing ratios of all species are efficiently quenched in and below the region of temperature inversion. Therefore, the decrease in mixing ratios is dictated by thermochemistry in this hot environment at the region of thermal inversion. For all DQ models and temperatures explored in this work we find that $\mathrm{CO}$ remains the dominant carbonbearing molecule as it is efficiently formed by thermochemical processes and barely affected by either chemistry or photodissociation. However, for the $\mathrm{T} 1$ case the concentration of $\mathrm{CH}_{4}$ becomes comparable to that of $\mathrm{CO}$ for the $\mathrm{HJ}$ orbiting cool G2 and $\mathrm{K} 0$ stars. This is because EQ renders similar mixing ratio values at those temperatures, and the XUV flux emitted from these stars is very weak in our photospheric models to dissociate these species (see bottom right plots in Figs. 7 and 8). A strong 

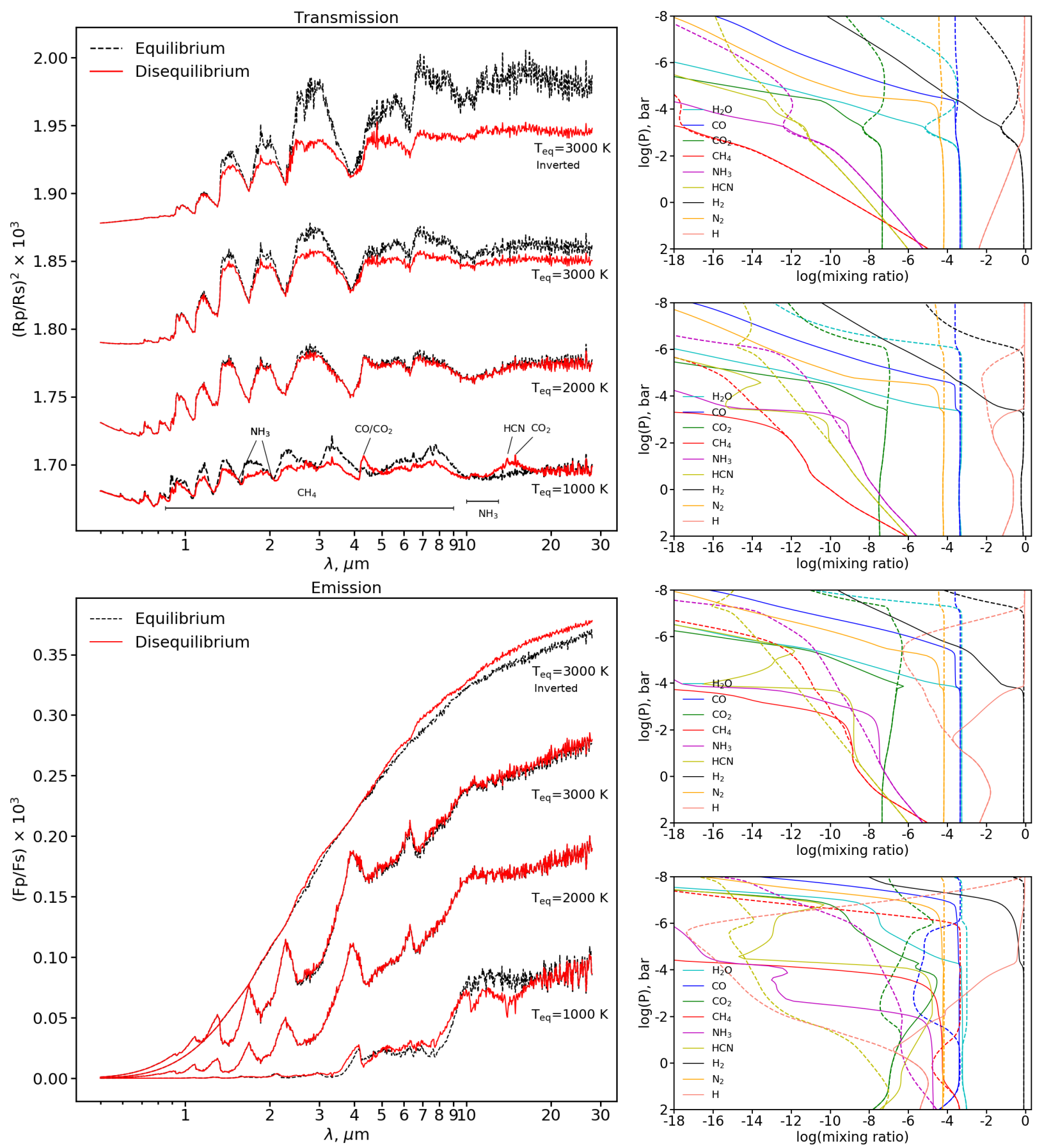

Fig. 5. Predicted transmission (top left) and emission (bottom left) spectra calculated for the Jupiter size planet around an A0 star. From bottom to top, the spectra predicted from the four different atmospheric structures are shown. The lines denoted as "Inverted" correspond to the calculations assuming analytical T-P profile with temperature inversion (see text for details). The spectra were binned with the spectral resolution $R=\lambda / \Delta \lambda=200$. The transmission spectra were shifted vertically for better representation. The mixing ratios corresponding to the above-mentioned atmospheric structures are shown in the second column of the figure (from bottom to top). In all plots, the spectra and mixing ratios calculated from the equilibrium and nonequilibrium chemistry are shown with dashed and solid lines, respectively. Disequilibrium molecules responsible for absorption bands in the emission spectrum for the T1 case are explicitly labeled in the top left plot.

UV flux is needed to noticeably distort the concentration of $\mathrm{N}_{2}$ away from its EQ value, and this remains almost unchanged in mid and low altitudes for all spectral types except A0. Molecular hydrogen remains the most abundant species (except at very high atmospheric layers) for temperatures $T_{\text {eq }}<3000 \mathrm{~K}$ and in all spectral types. As temperatures increase (i.e., as the exoplanet orbits closer to the host star), concentration of neutral hydrogen rises rapidly because of an efficient thermal dissociation and photodissociation of hydrogen-bearing molecules. But also the concentration of $\mathrm{HCN}$ rises for $\mathrm{T} 1$ planet compared to the $\mathrm{EQ}$ calculations for every star considered in this work. This is in full agreement with previous studies by Moses et al. (2011) and Hobbs et al. (2019) for HD 209458b and HD 189733b, although their analysis only pertained to $\mathrm{G}$ and $\mathrm{K}$ stars and ours pertains to a broader range of star types. When the photochemistry is included in the calculations, it largely influences the distribution 

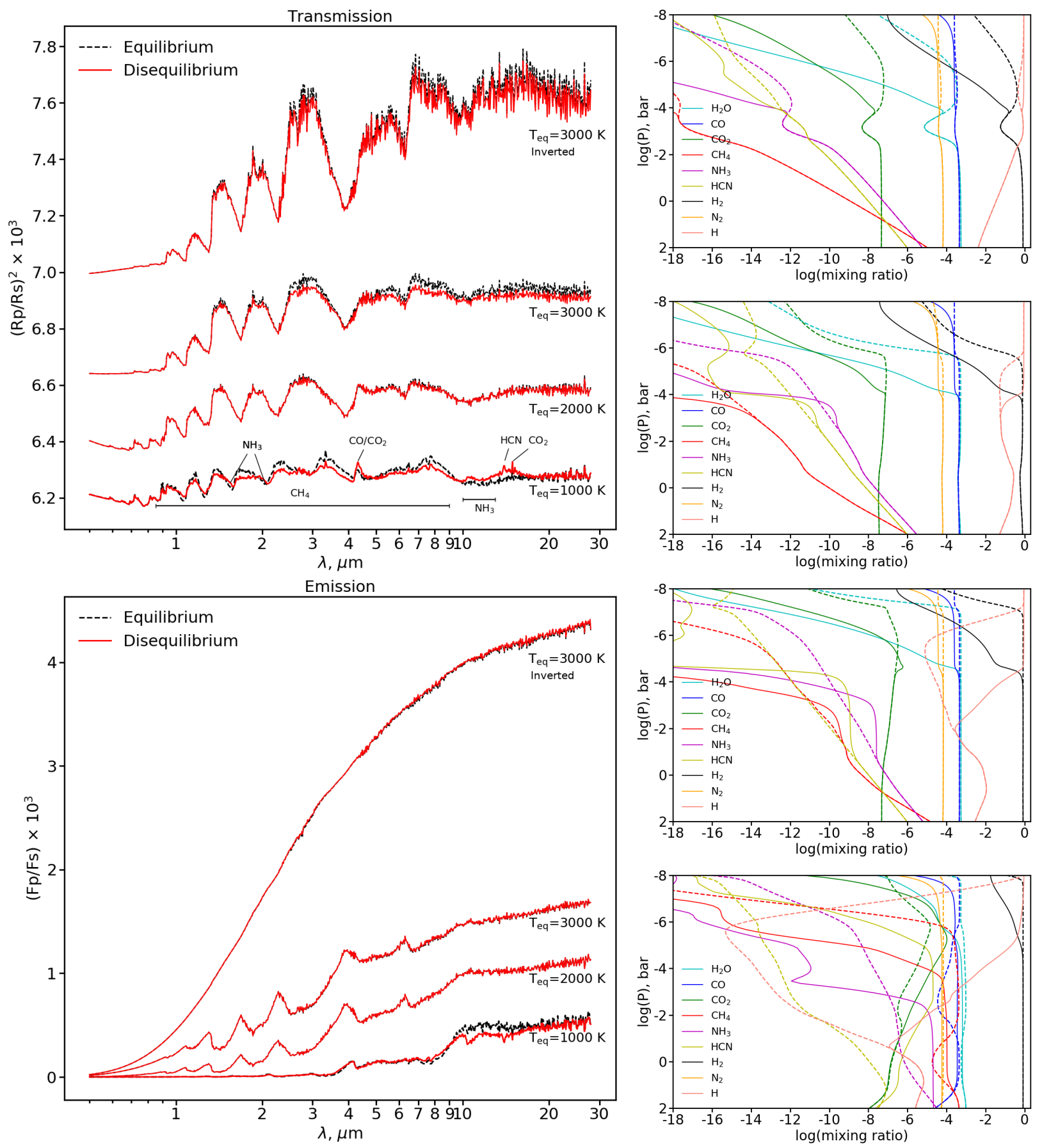

Fig. 6. Same as on Fig. 5, but for the planet around an F0 star.

of species in optically thin layers where the deposit of stellar XUV radiation is very efficient (and this effect is individual for each molecule). The two commonly used diffusion coefficients in 1D photochemical models (i.e., eddy and molecular diffusion coefficients) can determine the variation of mixing ratio profiles with pressure if the characteristic diffusion times are shorter than the chemical lifetimes. Otherwise, the vertical distribution of species is mainly controlled by photo- or thermochemical processes. We note that there might exist regions in which the associated lifetimes are similar and they can compete for shaping the mixing ratio profile. However, a detailed analysis of the prevalence of chemical and/or transport processes as a function of the star type, choice of the Kzz, and temperature of the exoplanetary atmosphere is beyond the scope of the current paper.

Finally, our calculations agree with previously reported conclusions that the impact of disequilibrium chemistry is stronger for cooler planets owing to the higher concentrations of photochemically active gases such as $\mathrm{CH}_{4}$ and $\mathrm{NH}_{3}$ (Madhusudhan et al. 2016; Moses 2014).

\subsection{Predicted transmission and emission spectra}

The left column of Figs. 5-8 shows transmission and emission spectra calculated using EQ and DQ mixing ratios discussed above. In our simulations the effect of spectral type is to provide 

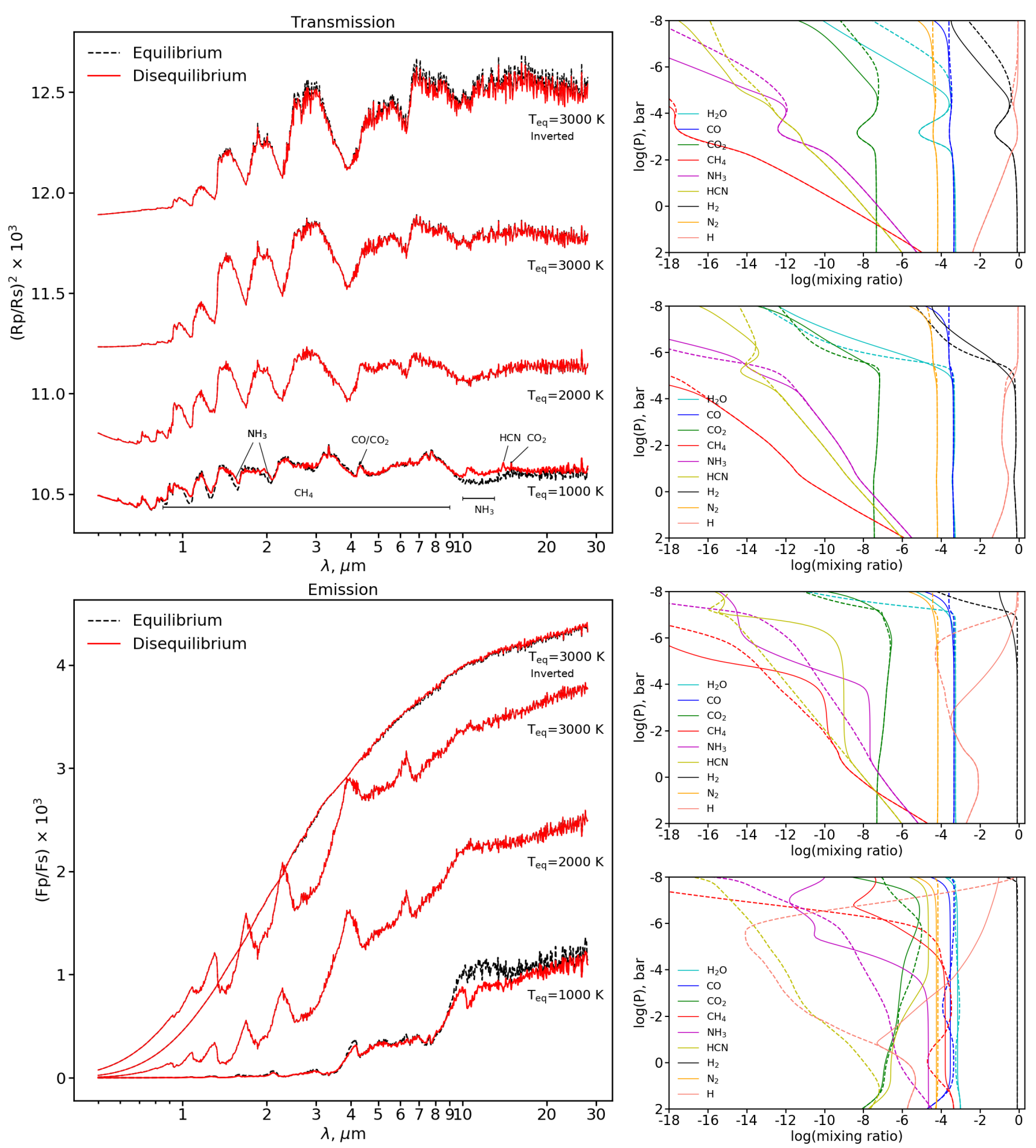

Fig. 7. Same as on Fig. 5, but for the planet around a G2 star.

nonthermal XUV radiation, which sets the rates of molecular photodissociation; the temperature, however, has effects on the transport processes and T-dependent chemical reactions. Hence, we detect most prominent changes between EQ and DQ models in atmospheric spectra for the hottest stellar type A0 and planets with $\mathrm{T} 1$ and T3-inverted temperature profiles. In Fig. 5 we highlight the spectral features of various chemical species across a range of wavelengths that are useful for identifying their signatures for the T1 case in transmission. For the T1 case, the DQ model predicts lower flux in the region between 10 and $20 \mu \mathrm{m}$ in the emission spectrum. This is the result of the increased $\mathrm{NH}_{3}$ concentrations and therefore stronger absorption in these wavelengths compared to EQ model. Absorption features at $10.4 \mu \mathrm{m}$ and $14 \mu \mathrm{m}$ that are seen in DQ models are due to $\mathrm{NH}_{3}$ and $\mathrm{HCN}$ lines, respectively. The increase of the spectral emission in DQ model compared to the EQ model between 3 and $4 \mu \mathrm{m}$, and between 7 and $9 \mu \mathrm{m}$ is due to photodissociation of $\mathrm{CH}_{4}$ and hence a weakening of the line absorption. In transmission the planet look larger in the wavelengths of increased opacity, that is, in the region between 10 and $20 \mu \mathrm{m}$ from the increased $\mathrm{NH}_{3}$ concentrations predicted by DQ model. At wavelengths shorter than that the transmission signal is decreased primarily as a result of 

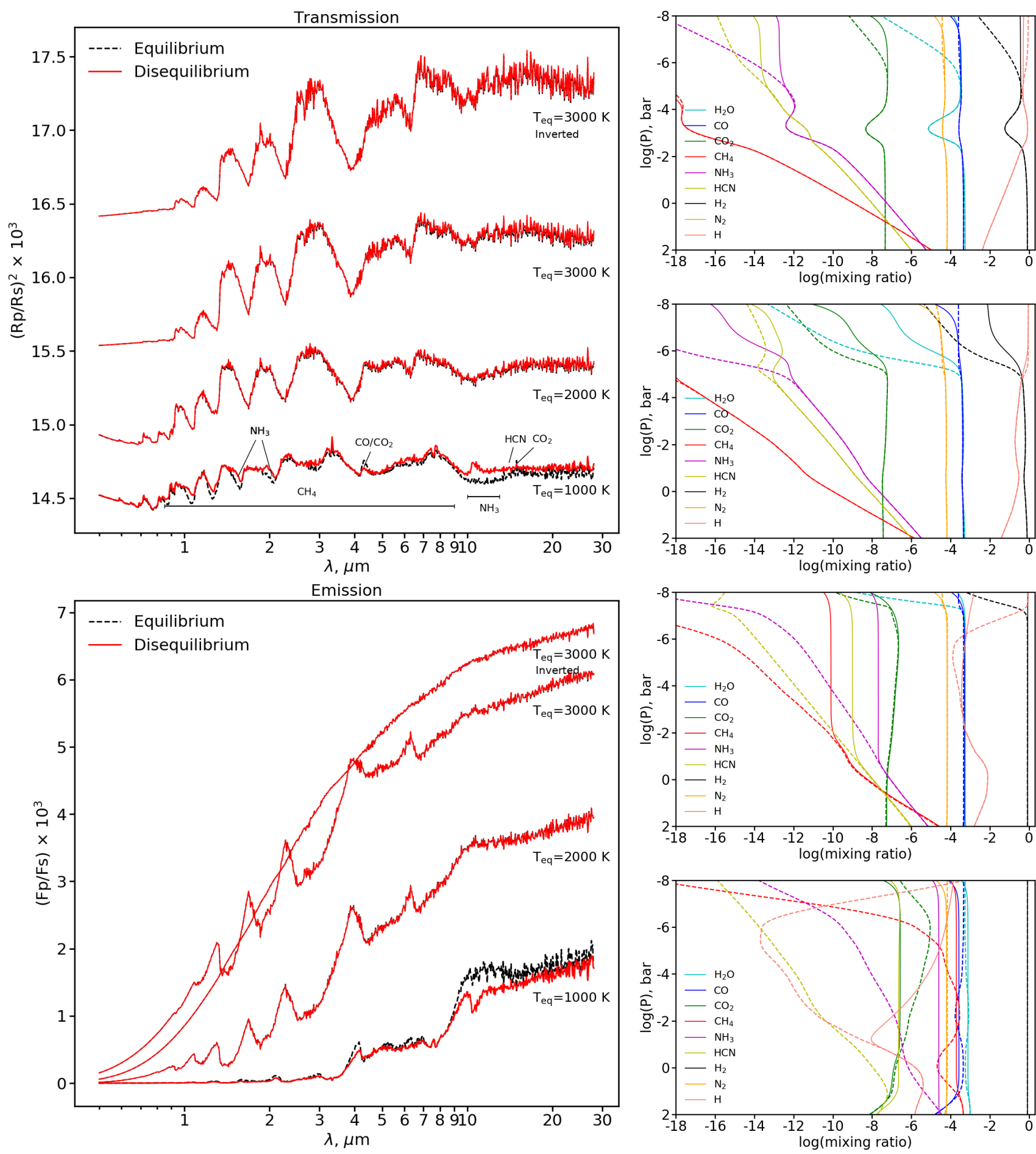

Fig. 8. Same as on Fig. 5, but for the planet around a K0 star.

a weaker absorption by $\mathrm{CH}_{4}$. A pronounced feature at $4.3 \mu \mathrm{m}$ is due to enhanced strengths of $\mathrm{CO}$ and $\mathrm{CO}_{2}$ bands. An increase in the transmission signal around 1.6 and $2.1 \mu \mathrm{m}$ is due to the increased absorption in $\mathrm{NH}_{3}$ bands located at these regions. We detect the same spectral behavior for the T1 planet around F0, $\mathrm{G} 2$, and $\mathrm{K} 0$ stars. We note that many expected features have been already identified in previous investigations (e.g., Moses et al. 2011; Venot et al. 2012; Blumenthal et al. 2018; Molaverdikhani et al. 2019a), and our study extends this analysis to a wider range of stellar spectral classes and atmospheric temperatures.
For the T2 planet both emission and transmission spectra show almost no changes between EQ and DQ models for all spectral types. In the case of a T3 planet we detect a noticeable decrease of the transmission amplitude, but only for A0 star; this is mainly, but not only, because of a strong photodissociation of $\mathrm{H}_{2} \mathrm{O}$. This is an interesting finding because it indicates that, for planets with $T_{\text {eq }} \approx 2000 \mathrm{~K}$, strong differences in mixing ratios that are driven by the radiation of the host star could not be robustly detected in the observed spectra. In the case of emission radiation, the photodissociation affects mixing ratios of 
atmospheric species at high altitudes that are transparent for the outgoing radiation and therefore do not significantly contribute to the emerging spectrum. Also, the abundance of $\mathrm{CH}_{4}, \mathrm{NH}_{3}$, and $\mathrm{CO}_{2}$ is very low even in the EQ model so that the changes in their mixing ratios introduced by photodissociation are not manifested in the final spectra. Strong photodissociation of $\mathrm{H}_{2}$ results in two orders of magnitude increase in the number density of neutral hydrogen atoms, but the corresponding opacity (due to Rayleigh scattering on $\mathrm{H}$ atoms and $\mathrm{H}^{-}$bound-free and free-free absorption) is not strong enough to drive changes in the predicted spectra either.

For the more realistic case of an UHJ with an inverted temperature profile (T3-inverted model) we observe strong differences between EQ and DQ models, which are driven by molecular photodissociation, but again only for A0 star. As a result, the amplitude of spectroscopic features due to, for instance, $\mathrm{H}_{2} \mathrm{O}$ and $\mathrm{CO}$ are strongly reduced in transmission. In emission, the DQ model emits more flux, thus making the planet look brighter. We note that the short wavelength part of the spectrum is not affected by disequilibrium chemistry and thus EQ and DQ models look the same for $\lambda<1 \mu \mathrm{m}$ in case of transmission and $\lambda<2 \mu \mathrm{m}$ in case of emission, respectively.

For the planets around F0, G2, and K0 the effect of disequilibrium chemistry is strongly reduced for T2, T3, and T3inverted cases. This is because of a weakening of the stellar XUV flux compared to A0 star, as well as very low mixing ratios of spectroscopically active molecules such as $\mathrm{CH}_{4}, \mathrm{NH}_{3}$, and $\mathrm{HCN}$ predicted by EQ and DQ models, which agrees with the predictions from previous studies (e.g., Madhusudhan et al. 2016).

\subsection{Impact of stellar activity}

To this end we used synthetic flux predicted by models of stellar photospheres to compute disequilibrium mixing ratios with VULCAN ignoring the activity-driven enhancement of XUV radiation. As we have noted before, stars with convective envelopes generate magnetically driven activity that manifests itself as an enhanced XUV radiation due to nonthermal atmospheric heating. Stars also undergo changes in their activity level as they age. Thus, in this section we investigate the impact of these two effects on the chemical structure of the atmosphere of our test planet.

First we compared predictions for the present Sun with its measured activity level and calculations for the G2 star without activity-driven XUV contribution via PHOENIX photospheric flux. As an example, Fig. 9 illustrates the amount of stellar flux that reaches various atmospheric depths as a function of wavelength for the T2 case. It is seen that most of the XUV flux of the modern Sun is efficiently absorbed at very high altitudes. In this particular $\mathrm{T} 2$ case the radiation with wavelengths longer than about $200 \mathrm{~nm}$ is capable of reaching very deep atmospheric layers, while flux at shorter wavelengths is fully absorbed at layers above $10^{-6}$ bar. As a result, we detect the strongest changes in species mixing ratios (compared to the equilibrium calculation) in these high altitudes, as shown in the right panel of Fig. 9. For the hottest T3 planet we find the same distribution of the absorbed radiation, but the XUV radiation reaches $\sim 10^{-5}$ bar. Because mixing ratios remain unaffected by stellar radiation at mid- and deep atmospheric layers, we detect almost no changes in the predicted transmission and emission spectra. We thus conclude that we must always account for the stellar activity contribution to the XUV flux in order to capture important changes in species concentrations of planets orbiting stars with convective envelopes. Strong photodissociation at these high altitudes increases concentrations of light species and thus enhances atmospheric erosion (Johnstone et al. 2019, e.g.). However, normal photospheric stellar models can still be used to predict planetary atmosphere spectra, at least in cases when the parent star is not at its strongest activity state.

Next, we analyzed the impact of enhanced stellar activity on our test planet assuming that it orbits the present Sun with its measured XUV flux and around the Sun when it was $100 \mathrm{Myr}$ young. At that young age the XUV flux of the Sun was maximal, thus driving the strongest changes in the atmospheric chemistry, compared in Fig. 10. We find that when activity is included, the $\mathrm{CO}$ still remains the dominant carbon-bearing molecule for all planetary temperatures considered owing to low photodissociation efficiency. That is, even very high XUV and Ly $-\alpha$ radiation are not able to photodissociate $\mathrm{CO}$ in lower and middle atmospheric layers. There is only enough stellar flux tot finally trigger dissociation of $\mathrm{CO}$ at high altitudes. For the $\mathrm{T} 1 \mathrm{case}$, the number density of $\mathrm{CH}_{4}$ also becomes relatively high in altitudes up to about $10^{-4}$ bar. Generally, for all planets we observe a strong photodissociation of molecules at high altitudes of the planet caused by increased stellar activity at dissociating wavelength ranges. This effect is more noticeable for planets closer to the host star (i.e., T2, T3 and T3-inverted cases). For the T3 and T3-inverted cases molecules $\mathrm{CH}_{4}$ and $\mathrm{HCN}$ show noticeably variable profiles at altitudes above $10^{-4}$ bar, with even increased concentrations in narrow pressure region.

Despite large changes in molecular mixing ratios between modern and young Sun calculations, we find no particular features in the transmission and emission spectra that could be used to estimate the age and therefore activity state of the host star (not shown here). Only the amplitude of the spectra is modified because the young Sun was $30 \%$ dimmer and $12 \%$ smaller compared to its present values.

\subsection{Constraining disequilibrium processes with future missions}

As shown above, the DQ models for HJs with $T_{\text {eq }}=1000 \mathrm{~K}$ produce characteristic strong features in transmission and emission spectra that clearly distinguish them from EQ models. These features could be studied with future space missions and the detection or, alternatively, non-detection of these features can provide strong constrains for modern photokinetic models.

To provide estimates of the observability of photochemically produced spectral features we used PANDEXO ${ }^{10}$ data simulation platform (Batalha et al. 2017) and carried out predictions for the James Webb Space Telescope (JWST) ${ }^{11}$. Figure 11 shows predicted emission and transmission spectra of our test planet around $\mathrm{A} 0$ and $\mathrm{G} 2$ stars for the $\mathrm{T} 1$ case. We chose these two stellar types because this is where the impact of disequilibrium chemistry is the strongest. We made predictions for the NIRSpec instrument with medium resolution mode (gratings G140M, $\mathrm{G} 235 \mathrm{M}$, and G395M) and MIRI instrument in low resolution mode (LRS). The large noise at wavelengths $\lambda>10 \mu \mathrm{m}$ is due to a dramatic drop in the MIRI-LRS throughput (Natasha Batalha, priv. comm.). The realistic noise floor for JWST instruments will not be known until after the instrument is operational, and similar to previous studies (Batalha et al. 2017; Greene et al. 2016) we assumed this value to be 20 and 50 ppm for the NIRSpec and MIRI-LRS modes, respectively. This noise floor was added as a quadrature to the photon noise generated by PANDEXO to obtain

\footnotetext{
10 https://natashabatalha.github.io/PandExo

11 https://www.jwst.nasa.gov
} 

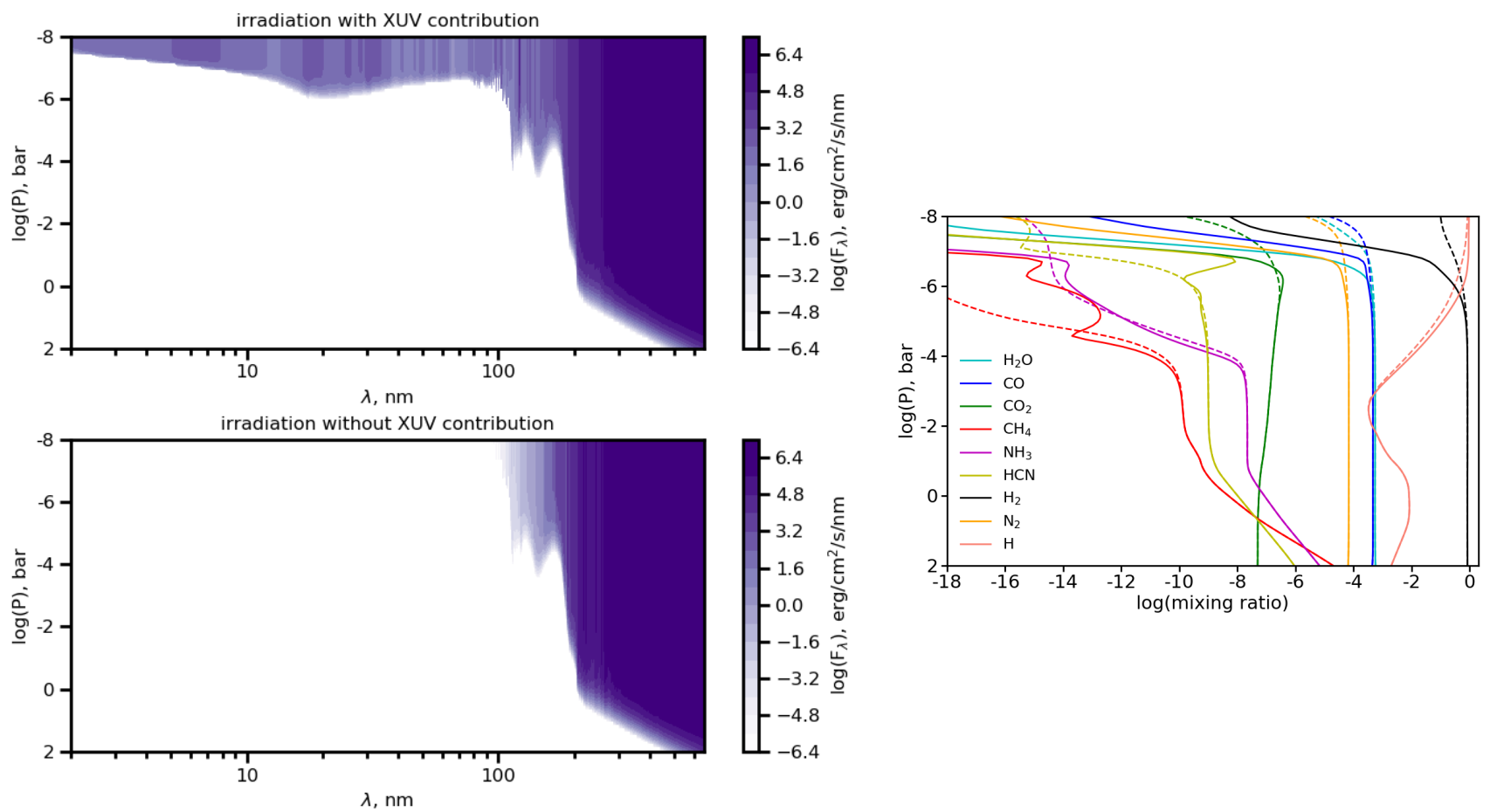

Fig. 9. Absorption of stellar flux in the atmosphere of a planet with $T_{\text {eq }}=2000 \mathrm{~K}$ as a function of wavelength (left panel) calculated using observed solar radiation (top panel) and stellar flux without an activity-driven XUV contribution, as predicted by photospheric PHOENIX model (bottom panel). The corresponding mixing ratios are shown in the right panel (dashed line - using PHOENIX flux, solid line - using observed solar flux).
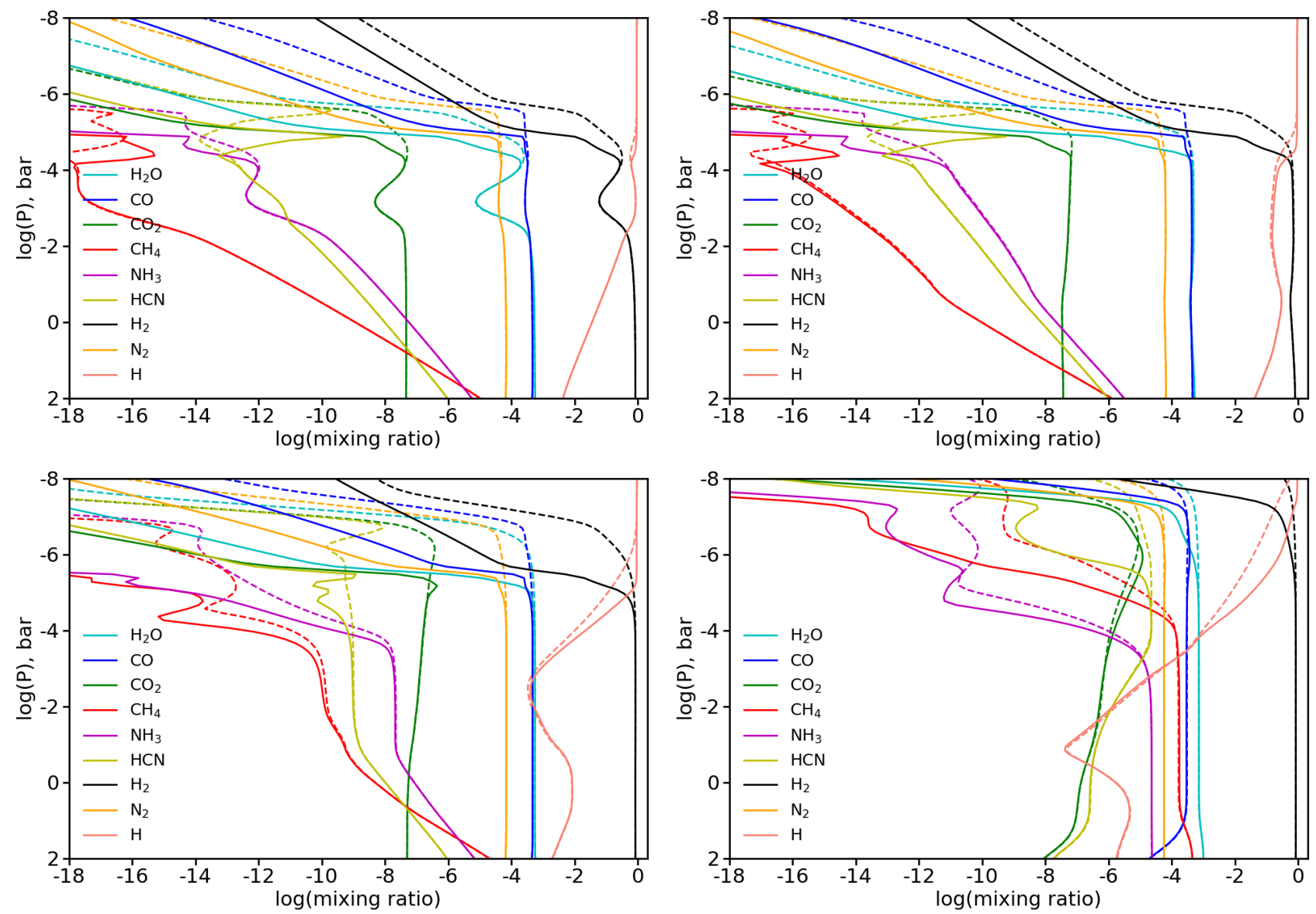

Fig. 10. Volume mixing ratios in the atmosphere of a Jupiter size planet around present (dashed lines) and 0.1 Gyr young Sun (solid lines) for the planet with equilibrium temperatures of $T_{\mathrm{eq}}=3000 \mathrm{~K}$ with temperature inversion (top left), $T_{\mathrm{eq}}=3000 \mathrm{~K}$ (top right), $T_{\mathrm{eq}}=2000 \mathrm{~K}$ (bottom left), and $T_{\mathrm{eq}}=1000 \mathrm{~K}$ (bottom right). 

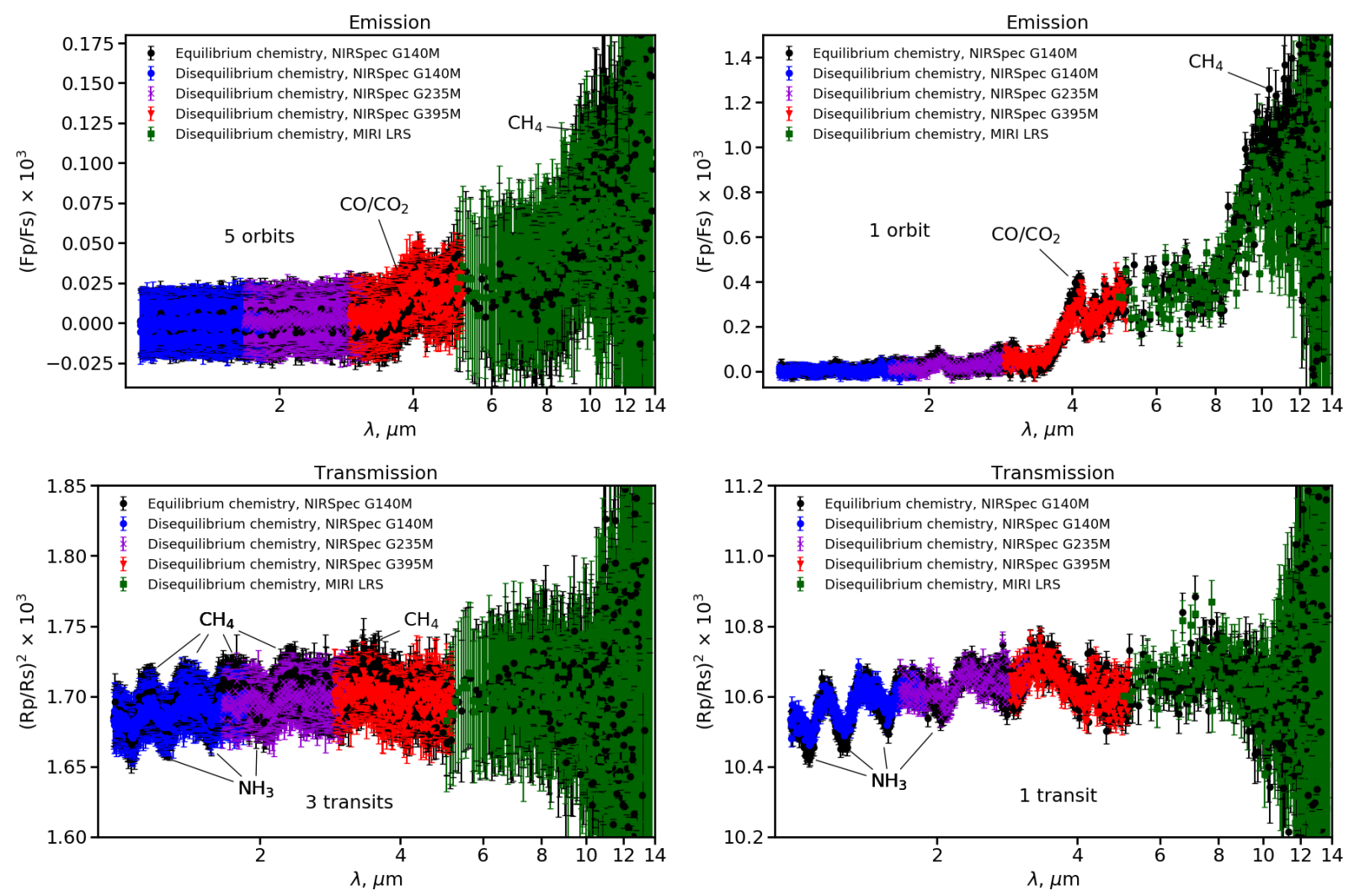

Fig. 11. Simulated emission (top row) and transmission (bottom row) spectra for a HJ planet with $T_{\text {eq }}=1000 \mathrm{~K}$ orbiting A0 (left) and G2 (right) stars using NIRSpec and MIRI instruments with JWST. The molecular features produced by photokinetic models that could be unambiguously detected with corresponding number of transits are explicitly indicated.

final errors on simulated data. We further binned the spectra to the resolution of $R=\lambda / \Delta \lambda=300$ to boost the signal-to-noise ratio $(\mathrm{S} / \mathrm{N})$ in each channel (excluding noise floor). The stars were assumed to have $K$-band magnitude $5^{\mathrm{m}}\left(\lambda_{\text {ref }}=2.22 \mu \mathrm{m}\right)$. The transit duration was 4 and $15 \mathrm{~h}$ for the $\mathrm{G} 2$ and $\mathrm{A} 0$ cases, with planet orbital periods being 8 and $114 \mathrm{~d}$, respectively. The total integration time for a single transit/orbit (without overheads) was 8 and $12 \mathrm{~h}$ for $\mathrm{G} 2$ stars and 30 and $45 \mathrm{~h}$ for $\mathrm{A} 0$ star in transmission and emission, respectively. We then adjusted the number of corresponding orbits/transits needed to reach a desired spectroscopic accuracy to robustly detect features caused by disequilibrium processes.

We found out that the noise floor is already easily reached in many wavelength channels in just a few orbits/transits (especially in G140M and G235M wavelengths, and to a lesser extent in $\mathrm{G} 395 \mathrm{M}$ ), and thus the binning of the data to even lower resolution does not help to decrease the final errors. The noise floor is never reached in MIRI-LRS within the integration time considered in our simulations, and the intrinsic instrument performance thus sets the limit on the final errors. However, the detection of features due to disequilibrium processes would still be possible thanks to a large number of wavelengths that can be observed simultaneously. We estimate the detectability of spectroscopic features by using $\chi^{2}$ test between original spectra of EQ and DQ models and simulated DQ observations, respectively. The corresponding values of $\chi^{2}$ were only computed within spectral intervals affected by disequilibrium processes.

Our estimates show that it would be challenging to spectroscopically observe photochemical signatures in the atmosphere of a planet orbiting A0 star because the star-to-planet size ratio as well as the flux contrast is very unfavorable at near-infrared wavelengths. In emission observations, five orbits would be needed to detect the effect of disequilibrium chemistry using the $\mathrm{CO} / \mathrm{CO}_{2}$ feature at $4.3 \mu \mathrm{m}$ above $3 \sigma$ threshold. In transmission, many disequilibrium effects could be seen with three transits, including weak $\mathrm{NH}_{3}$ features (see annotations on Fig. 11). On the other hand, the decrease in the transmission amplitude at $1.8 \mu \mathrm{m}$ and $2.23 \mu \mathrm{m}$ due to the dissociation of $\mathrm{CH}_{4}$ could already be detected with even a fewer number of orbits. We note, however, that obtaining transmission spectrum even for this very bright $\mathrm{A} 0$ star takes $30 \mathrm{~h}$ of integration time, which is split between $15 \mathrm{~h}$ in- and 15 h out-of-transit observations.

For the $\mathrm{G} 2$ star case, the robust detection of the $\mathrm{CO} / \mathrm{CO}_{2}$ feature at $4.3 \mu \mathrm{m}$ and $\mathrm{CH}_{4}$ at $11 \mu \mathrm{m}$ above $3 \sigma$ level requires only one orbit in emission observations, as shown in the top right plot in Fig. 11. In transmission, one transit is needed to detect $\mathrm{NH}_{3}$ features, but detecting the $\mathrm{CO} / \mathrm{CO}_{2}$ feature at $4.3 \mu \mathrm{m}$ and $\mathrm{NH}_{3}$ absorption at $10 \mu \mathrm{m}$ remain challenging and will require a larger number of transits for a robust analysis.

The HCN feature seen at $14 \mu \mathrm{m}$ for A0 case can potentially be detected with the JWST/MIRI instrument (channel 3, wavelength rage 11.53-18.03 $\mu \mathrm{m}$ ) using the medium-resolution integral field unit mode (MIRI MRS). Because the current version of the PANDEXO does not support this observing mode, we could not calculate the expected noise level and thus the required observing time. However we notice that amplitude of the HCN band is relatively strong compared to other photochemically produced features and thus could be addressed with JWST.

Although the difference between EQ and DQ models is large for the T3-inverted cases of an A0 star, it would be fairly difficult to study photochemical processes because the predicted changes 

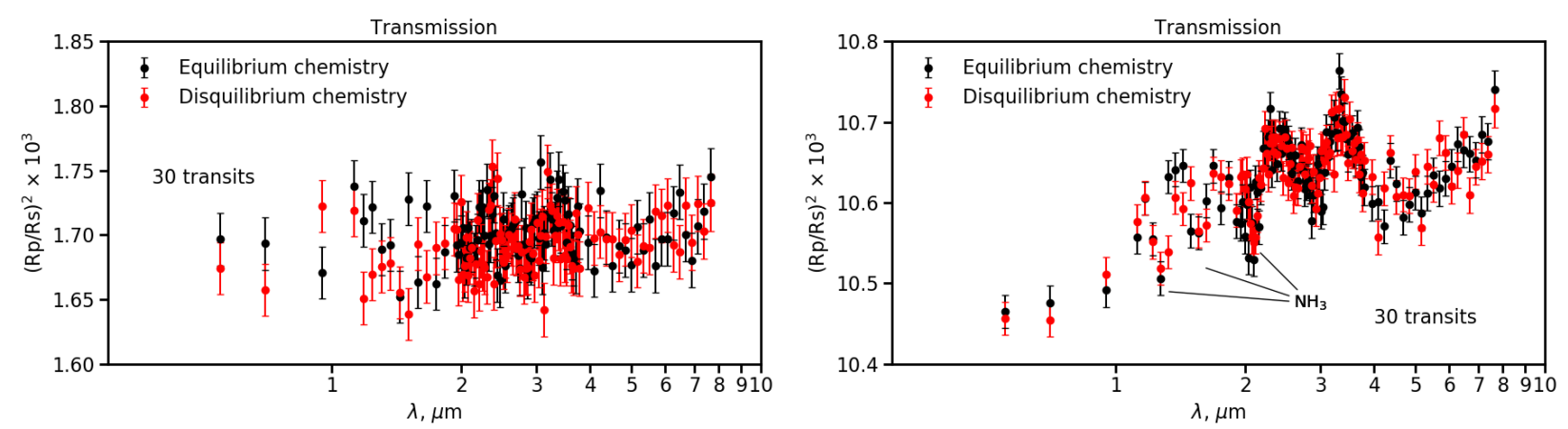

Fig. 12. Simulated ARIEL transmission spectra of a $T_{\text {eq }}=1000 \mathrm{~K}$ planet orbiting A0 (left) and G2 (right) star.

in the transmission spectra are the same in a wide wavelength range.That is, the DQ model looks just like the EQ model with decreased transmission amplitude (see Fig. 5). Nevertheless, with accurate enough observations this decrease in the amplitude of spectral features could also be studied by comparing flux at short and long wavelengths or by analyzing the amplitude of the molecular bands between 3 and $4 \mu \mathrm{m}$ and 6 and $7 \mu \mathrm{m}$, respectively. From simulations listed above we conclude that the spectroscopic accuracy of JWST will be sufficient to study strong changes in the transmission amplitude in a wide wavelength range cased by disequilibrium processes in case of inverted temperature profile (T3-inverted case). However, more detailed calculations are still needed to compute electron number densities and thus $\mathrm{H}^{-}$opacity self-consistently. Overall we find that JWST/NIRSpec will be just the right instrument to study individual details in spectra of extrasolar planets owing to its sufficient spectral resolution and wide wavelength coverage.

Low-resolution spectroscopy will also be available at the Atmospheric Remote-sensing Infrared Exoplanet Large-survey space mission (ARIEL) ${ }^{12}$ planned by ESA for 2028 (Tinetti et al. 2018). The instrument will have few photometric filters between 0.5 and $1 \mu \mathrm{m}$, and two spectroscopic options with resolving power $R=100$ between 1.95 and $3.95 \mu \mathrm{m}$ and $R=30$ between 3.95 and $7.8 \mu \mathrm{m}$, thus covering a spectral region in which the signatures of disequilibrium chemistry can be studied. We used the latest ARIEL noise simulator (L. Mungai and E. Pascale, priv. comm.) to estimate the noise level in instrument pass bands. As an example, Fig. 12 shows the transmission spectra (with random noise added) of our test planet around A0 and G2 stars assuming the same transit duration and stellar magnitude as above for the JWST. Unfortunately, the relatively small number of available wavelengths and the small size of the telescope makes it virtually impossible to constrain disequilibrium processes because the $3 \sigma$ detection threshold is almost never reached. For A0 star, even after 30 transits the DQ model deviates only by $1 \sigma$ from the EQ model with the noise floor dominated. With the same 30 transits we reach on average a $3 \sigma$ threshold for the G2 star, but more transits would not help as a result of hitting the noise floor. We note that studying disequilibrium processes might still be possible for planets orbiting low-mass stars.

\section{Discussion}

\subsection{Consistency between atmospheric temperature and mixing ratios}

In our calculations of the impact of disequilibrium chemistry on the observed emission and transmission spectra of HJs we used

12 https://arielmission. space/ the temperature structure generated with HELIOS but assuming chemical equilibrium concentrations. The latter were calculated with FASTCHEM and were used to compute continuum and line opacity needed for HELIOS. However, as initial equilibrium mixing ratios are changed by photochemistry and kinetics, they also change local opacity and therefore local temperature which, in turn, changes mixing ratios. This means that in our approach the planetary T-P structure is inconsistent with the results of disequilibrium chemistry calculations. For instance, Drummond et al. (2016) found that for such well-known planets as HD $189733 \mathrm{~b}\left(T_{\mathrm{eq}}=1200 \mathrm{~K}\right)$ and HD $209458 \mathrm{~b}\left(T_{\mathrm{eq}}=1500 \mathrm{~K}\right)$ the effect of not including consistent calculations between chemistry and temperature leads to an overestimation of the impact of disequilibrium chemistry on the predicted emission spectra. This happens because once temperature iteration is introduced in the disequilibrium chemistry algorithm, the model P-T structure adapts to the evolution of mixing ratios to maintain the global energy balance in the planetary atmosphere (which is set by the corresponding $T_{\text {eq }}$ of the planet). Therefore, even though the final mixing ratio profiles and P-T distribution look different from their initial equilibrium states, the predicted emission spectra does not deviate very much from equilibrium calculations. It does however contain some distinct features and thus disequilibrium chemistry must be taken into account for accurate interpretations. For instance, the $\mathrm{NH}_{3}$ feature at $10 \mu \mathrm{m}$ is present in both purely photochemical and self-consistent photochemical models (see Fig. 7 in Drummond et al. 2016). This feature is also prominent in our simulations of the emission spectra of the T1 planet and thus should be robustly detected in future observations.

\subsection{Atmospheric opacity}

In our calculations of $\mathrm{H}^{-}$opacity we used the equations given in John (1988) for bound-free and free-free processes. These equations require a knowledge of $\mathrm{e}^{-}$and $\mathrm{H}$ I number densities. Unfortunately, at its current stage VULCAN does not account for the ionization of atomic species. Therefore, we used the concentrations of electrons calculated by FASTCHEM and concentrations of $\mathrm{H}$ I from VULCAN to compute $\mathrm{H}^{-}$opacity. We do not expect this inconsistency to affect our results much. At least it should not be an issue for $\mathrm{T} 1$ and $\mathrm{T} 2$ cases in which the atmospheric temperatures are too low for the $\mathrm{H}^{-}$and $\mathrm{He}^{-}$opacity to play any significant role. However, for T3 and T3-inverted cases, a more accurate calculation of $\mathrm{e}^{-}$concentrations is desired, especially in dense and hot layers around photosphere and below.

\subsection{Stellar activity: XUV radiation}

In this work we explicitly address the impact of stellar activity on the planetary spectra of a G2 star using observed XUV flux 
of the modern Sun as a proxy. We then additionally investigate the changes in mixing ratios and spectra of a young Sun of age 0.1 Gyr. This is the youngest age available from the study by Claire et al. (2012) and at that age the Sun had the highest XUV flux so that the changes in photochemistry were the strongest. We note that, according to Tu et al. (2015), sun-like stars with ages between 20 and 300 Myr maintain very high X-ray flux, which is almost constant with time and does not depend on stellar rotation rate and hence the age. After $0.3 \mathrm{Gyr}$ the activity in a majority of sun-like stars begins to desaturate and their X-ray flux starts to decay with time as the rotation rate keeps decreasing because of magnetic braking. Thus, in principle we could have chosen any parameters for the young Sun within this time interval but this would not have changed the general trends in our results.

One important caution needs still to be made when addressing activity in young suns. Observations of stellar clusters demonstrated that sun-like stars are born with a rather wide range of rotation periods (e.g., Soderblom et al. 1993) and it is not until about 1 Gyr that they all presumably converge to the same rotation period of about ten days (Tu et al. 2015). Because the age at which the stellar activity stars desaturate depends on the initial rotation period, sun-like stars that are younger than 1 Gyr could still have very different XUV flux, depending whether they were born as fast or slow rotators (Tu et al. 2015). That means that, for example, for the Sun it is currently impossible to predict the true value of its XUV flux when it was young because we do not know how fast the young Sun was rotating in a first place. However, this is not an issue in our study because we analyzed a hypothetical gas giant planet around a young sun-like star and we assumed that this star was very active in its early evolution stages with best-to-date estimates of the expected XUV radiation taken from Claire et al. (2012). It is obvious that studying the chemical evolution of atmospheres of real planets must account for accurate knowledge of the activity evolution of their hosts.

In this study we did not consider the impact of activity for the planets orbiting K0 star. Indeed, as was mentioned above, all stars with convective envelopes generate nonthermal XUV emission similar to our Sun. What is different from sun-like stars, however, is the less efficient magnetic braking of $\mathrm{K}$ and $\mathrm{M}$ stars because of their small sizes (Reiners \& Mohanty 2012). As a result, $\mathrm{K}$ and $\mathrm{M}$ stars can maintain strong activity levels for a much longer time compared to sun-like stars with a potentially stronger long-term impact on atmospheric chemistry. Moreover, M dwarfs are known to generate the strongest magnetic fields among all stars with convective envelopes (Shulyak et al. 2017), thereby resulting in extreme magnetic heating of their chromospheres and coronae. For instance, the ratio of X-ray to total luminosity of M dwarfs are 10-100 times higher than those of the present day Sun (Poppenhaeger et al. 2010), whereas their bolometric luminosity are 10 to 1000 times smaller. Reconstructing XUV spectrum of these stars is a difficult task. Nevertheless, for a dozen of planet host targets the combined X-ray, UV, visual, and infrared data exist and are available from, for example, the MUSCLES survey ${ }^{13}$ (France et al. 2016; Youngblood et al. 2016; Loyd et al. 2016). These data could be used to compute realistic photochemical models of planets orbiting these small stars. However, we expect that the activity of $\mathrm{K}$ stars affects the observed spectra similar to the case of a young Sun, although the amplitude of the effect could be stronger because of an increase in a relative X-ray luminosity and the fact the our test planet would be closer to the star (to maintain equilibrium temperatures that we assumed in this work).

13 https://archive.stsci.edu/prepds/muscles/
As stars spin down their chromospheric activity eventually fades away. But even in this case old stars with convective envelopes can still have non-zero X-ray emission, this time of nonmagnetic nature, which is called basal emission (Schrijver 1995; Schrijver et al. 1989) and can be a major source of high-energy photons that drive planetary atmospheres out of equilibrium, even if stellar dynamo is very weak or even absent.

\subsection{Stellar activity: winds}

Winds are another aspect of stellar activity that may have a strong impact on the atmospheric structure at high altitudes. Stellar winds carry high energetic particles that collide with atmospheric constitutes, dissociate and ionize them, and thus raise local atmospheric temperatures. Unless planets have strong magnetic fields that protect their atmospheres against stellar winds, the latter cannot be ignored, especially for active stars. Building realistic wind models for stars of various types and ages is very challenging because of many unknown parameters that often rely on very limited observations (see Leitzinger et al. 2014, and references therein). Nevertheless, some sophisticated wind models have been developed that account for outflows dominated by CMEs (Johnstone et al. 2015b,a) and/or 3D magnetohydrodynamic wind models that can take into account the observed properties of stellar magnetic fields (Vidotto et al. 2015).

Overall, it is seen that the impact of stellar activity on the atmospheric structure of planets must include many physical and chemical processes that are not trivial to model and often even estimate. In this work we limited ourselves to only stellar XUV radiation. Addressing activity impact in real stars would surely require consideration of all these processes to the best of our knowledge.

\subsection{Clouds and hazes}

In this work we ignored the possible contribution of clouds and hazes to the observed planetary spectra. It was shown that some HJs may have thick cloud decks at high enough altitudes that are probed with transmission spectroscopy (Sing et al. 2016). The effect of clouds would weaken spectroscopic features due to scattering absorption. This effect is largest at optical wavelengths and decays for longer wavelengths. Thus, the presence of clouds and hazes can mute the photochemically produced features of $\mathrm{NH}_{3}$ between 1 and $3 \mu \mathrm{m}$. Nevertheless, clouds are not expected to seriously affect spectra of planetary atmospheres at $\lambda>10 \mu \mathrm{m}$, except perhaps to condensate vibrational mode features, which can become prominent in the infrared (Wakeford \& Sing 2015); thus this favors the analysis of disequilibrium effects in that spectral domain.

\subsection{Three-dimensional atmospheric effects}

In this work, we considered a 1D photochemical model representative of mid-latitudes and globally averaged diurnal conditions. Also, the same temperature structure for the day and night sides of our test planet were assumed when predicting its spectra. In reality, however, chemistry in atmospheres of HJs could be strongly modified by the presence of large-scale circulations induced by the temperature difference between the day and night sides. One of the results of such mixing is to produce complex abundance distribution, which is homogenized longitudinally and thus quenched to the day side values. However, for some molecules, such as $\mathrm{CH}_{4}$, the abundance distribution can still have 
large variations over evening and morning limbs (Agúndez et al. 2012 , 2014). This demonstrates that $3 \mathrm{D}$ effects can have strong global impact on the atmospheric chemistry (Molaverdikhani et al. 2020; Drummond et al. 2018b,a). In emission observations, the effect of atmospheric winds may not be particularly important owing to a rather large flux contrast between day and night sides (e.g., Mendonça et al. 2018), however the thermal inversions in the upper atmospheric layers may result in noticeable differences in day and evening sides of the planet that could possibly alter its disk integrated spectrum (Agúndez et al. 2014). In transmission, to the contrary, the effect of circulations may play an important role in rendering the observed spectra because transmission observations probe the terminator region where the differences in atmospheric thermal structure and physicochemical processes determined by $T(p)$ are expected to be maximal and that, if ignored, could introduce biases in the retrieved atmospheric parameters (Caldas et al. 2019).

\subsection{Planetary parameters}

We considered only one case of a Jupiter size planet orbiting stars of different temperatures. Our intention was to study a general trend of changes in the predicted spectra caused by disequilibrium chemistry rather than to investigate particular details of the mixing ratio profiles as a function of planetary parameters. For instance, planets more massive than one Jupiter mass would have denser atmospheres and hence less efficient turbulent mixing. Also, the penetration depth of the XUV radiation would be smaller. Molaverdikhani et al. (2019b) recently carried out an extensive investigation of these effects.

\section{Summary}

In this work we investigate how disequilibrium chemistry impacts the observed spectra and molecular mixing ratios in the atmosphere of a HJ orbiting stars of various types. We also explicitly address the impact of stellar activity on the derived mixing ratios and spectra simulated at primary and secondary eclipses. We demonstrate how these processes cause different trends in the predicted spectra, and identify major factors that can effect interpretation of observations. The main conclusions of this work are summarized below.

Photodissociation by external stellar radiation and chemical kinetic drive mixing ratios far from their thermo-equilibrium values. This effect is stronger for planets orbiting hot stars because those generate stronger XUV fluxes compared to stars of later spectral types. For planets with equilibrium temperatures around $T_{\text {eq }}=1000 \mathrm{~K}$ the effect of disequilibrium chemistry for some molecules $\left(\mathrm{HCN}, \mathrm{NH}_{3}, \mathrm{CH}_{4}\right)$ can be seen even in optically thick layers around 1 bar and deeper, in agreement with previous studies (e.g., Moses 2014).

In all cases considered the $\mathrm{CO}$ remains the dominant carbonbearing molecule in the atmosphere. However, for planets with $T_{\text {eq }}=1000 \mathrm{~K}$ that orbit $\mathrm{G} 2$ and $\mathrm{K} 0$ stars the concentration of $\mathrm{CH}_{4}$ becomes comparable to that of $\mathrm{CO}$ at $P<0.1$ mbar, whereas at higher altitudes, a smaller XUV radiation from the $\mathrm{K} 0$ star allows for rather similar $\mathrm{CO}$ and $\mathrm{CH}_{4}$ profiles throughout the atmosphere given the inefficient methane photodissociation.

We find that because of the effect of disequilibrium chemistry on the emission a transmission spectra is better observed for planets with $T_{\text {eq }}=1000 \mathrm{~K}$ for all stellar spectral types, thus favoring planets with relatively cool temperatures for the in-depth analysis of disequilibrium effects (Madhusudhan et al. 2016). We find, in addition, that disequilibrium chemistry has strong impact on the emission and transmission spectra of ultra hot planets with temperature inversion at high altitudes and that orbit Atype stars only. For planets with $T_{\text {eq }}=2000 \mathrm{~K}$ the observational consequences of disequilibrium chemistry on the spectra are not evident.

In transmission, the most prominent features due to disequilibrium chemistry are the $\mathrm{NH}_{3}$ bands at $1,1.3,1.5$, and $2 \mu \mathrm{m}$, as well as the strengthening of the $\mathrm{CO} / \mathrm{CO}_{2}$ absorption at $4.3 \mu \mathrm{m}$ and $\mathrm{NH}_{3}$ absorption at $10 \mu \mathrm{m}$. We also notice a weakening of the $\mathrm{CH}_{4}$ band at $3.3 \mu \mathrm{m}$. Finally, in our calculations the disequilibrium chemistry leads to the appearance of the narrow $\mathrm{HCN}$ absorption at $14 \mu \mathrm{m}$, which never shows up in equilibrium models. The appearance of this HCN feature has been noted in some previous works (e.g., Moses et al. 2011) and its observation could potentially provide a benchmark test for modern disequilibrium models.

The enhanced XUV radiation plays a crucial role in shaping mixing ratios of most atmospheric species around stars with chromospheric activity. The strongest impact of XUV radiation is expected in planets orbiting stars of young ages that have the highest level of chromospheric activity. However, even in the case of the present Sun with its known and relatively low activity level, accounting for the true nonthermal XUV flux in disequilibrium calculations is needed to predict realistic concentrations of species at high atmospheric layers. On the other hand, in HJs orbiting stars similar to our Sun (in term of temperature and age), the stellar XUV flux does not penetrate in line-forming regions of planetary atmospheres and thus does not affect their spectral appearance much.

Spectra from future facilities with spectroscopic capabilities in infrared promise to identify disequilibrium chemical constituents and their underlying kinetic controlling mechanisms. Using the NIRSpec and MIRI instrument onboard of the JWST it will be possible to detect and analyze photochemically induced changes in the observed spectra of hot exoplanets, in agreement with estimates by Blumenthal et al. (2018). In particular, the typical changes that we predict in this study are observed with sufficiently low noise level in only one transit around a sun-like star with a $K$-band magnitude of $5^{\mathrm{m}}$. The future ESA mission ARIEL will also have spectroscopic capabilities. Although the detection of disequilibrium chemistry could in principle be possible within $3 \sigma$ confidence interval in HJs around G-type stars, the robust analysis would likely be not possible mainly because of the low spectroscopic resolution of the instrument. Planethunting missions such as TESS ${ }^{14}$, PLATO $^{15}$, and CHEOPS ${ }^{16}$ are expected to significantly increase the number of exoplanets that orbit bright stars that will be accessible for detailed atmospheric characterizations.

Similar to many previous works, we ignored the effect of disequilibrium chemistry on the temperature structure of planetary atmospheres. This effect is expected to be important at least in some temperature regimes (Drummond et al. 2016) and should be taken into account in future works.

Acknowledgements. Authors thank the anonymous referee for insightful comments and suggestions to the manuscript. Authors also wish to thank Matej Malik for his help with HELIOS and HELIOS-K codes and Natasha Batalha for her help with PandExo simulation package. The estimates of exposure times for the ARIEL mission were kindly provided by Lorenzo Mungai and Enzo Pascale. We additionally thank Bandow Bernhard for the help installing CUDA and Paul Hartogh for helpful discussions. We acknowledge the support of the DFG priority program SPP-1992 "Exploring the Diversity of Extrasolar Planets" (DFG PR 36

\footnotetext{
14 https://heasarc.gsfc.nasa.gov/docs/tess/

15 http://sci.esa.int/plato/

16 http://sci.esa.int/cheops/
} 
24602/41). LML acknowledges the financial support from the State Agency for Research of the Spanish MCIU through the "Center of Excellence Severo Ochoa" award SEV-2017-0709, and from the research projects ESP2016-76076-R and PGC2018-099425-B-I00. We also acknowledge the use of electronic databases SIMBAD and NASA's ADS.

\section{References}

Abel, M., Frommhold, L., Li, X., \& Hunt, K. L. C. 2011, in 66th International Symposium On Molecular Spectroscopy, EFC07

Abel, M., Frommhold, L., Li, X., \& Hunt, K. L. C. 2012, J. Chem. Phys., 136, 044319

Agúndez, M., Venot, O., Iro, N., et al. 2012, A\&A, 548, A73

Agúndez, M., Parmentier, V., Venot, O., Hersant, F., \& Selsis, F. 2014, A\&A, 564, A73

Arcangeli, J., Désert, J.-M., Line, M. R., et al. 2018, ApJ, 855, L30

Bakos, G. Á., Bayliss, D., Bento, J., et al. 2018 AJ, submitted, [arXiv:1812.09406]

Batalha, N. E., Mandell, A., Pontoppidan, K., et al. 2017, PASP, 129, 064501

Bayliss, D., Gillen, E., Eigmüller, P., et al. 2018, MNRAS, 475, 4467

Birkby, J. L., Cappetta, M., Cruz, P., et al. 2014, MNRAS, 440, 1470

Blumenthal, S. D., Mandell, A. M., Hébrard, E., et al. 2018, ApJ, 853, 138

Borysow, A. 2002, A\&A, 390, 779

Borysow, A., \& Frommhold, L. 1989, ApJ, 341, 549

Borysow, A., Jorgensen, U. G., \& Fu, Y. 2001, J. Quant. Spectr. Rad. Transf., 68, 235

Caldas, A., Leconte, J., Selsis, F., et al. 2019, A\&A, 623, A161

Carbon, D., Gingerich, O. J., \& Latham, D. W. 1969, in Low-Luminosity Stars, ed. S. S. Kumar (London: Gordon \& Breach Science Publishers Ltd), 435

Casasayas-Barris, N., Pallé, E., Yan, F., et al. 2019, A\&A, 628, A9

Cauley, P. W., Wilson and Shkolnik, E. L., Llama, J., \& Lanza, A. F. 2019, Nat. Astron., 3, 1128

Christensen, U. R., Holzwarth, V., \& Reiners, A. 2009, Nature, 457, 167

Claire, M. W., Sheets, J., Cohen, M., et al. 2012, ApJ, 757, 95

Dalgarno, A. 1962, in Spectral Reflectivity of the Earth's Atmosphere iii: the Scattering of Light by Atomic Systems (USA: Ft. Belvoir Defense Technical Information Center)

Debes, J. H., \& Jackson, B. 2010, ApJ, 723, 1703

Delrez, L., Santerne, A., Almenara, J. M., et al. 2016, MNRAS, 458, 4025

Drummond, B., Tremblin, P., Baraffe, I., et al. 2016, A\&A, 594, A69

Drummond, B., Mayne, N. J., Manners, J., et al. 2018a, ApJ, 869, 28

Drummond, B., Mayne, N. J., Manners, J., et al. 2018b, ApJ, 855, L31

Dwivedi, N. K., Khodachenko, M. L., Shaikhislamov, I. F., et al. 2019, MNRAS, 487, 4208

Fontenla, J. M., Linsky, J. L., Witbrod, J., et al. 2016, ApJ, 830, 154

France, K., Loyd, R. O. P., Youngblood, A., et al. 2016, ApJ, 820, 89

García Muñoz, A. 2007, Planet. Space Sci., 55, 1426

Greene, T. P., Line, M. R., Montero, C., et al. 2016, ApJ, 817, 17

Grimm, S. L., \& Heng, K. 2015, ApJ, 808, 182

Güdel, M., Guinan, E. F., \& Skinner, S. L. 1997, ApJ, 483, 947

Guillot, T. 2010, A\&A, 520, A27

Harris, G. J., Tennyson, J., Kaminsky, B. M., Pavlenko, Y. V., \& Jones, H. R. A. 2006, MNRAS, 367, 400

Hartman, J. D., Bayliss, D., Brahm, R., et al. 2015, AJ, 149, 166

Heays, A. N., Bosman, A. D., \& van Dishoeck, E. F. 2017, A\&A, 602, A105

Hobbs, R., Shorttle, O., Madhusudhan, N., \& Rimmer, P. 2019, MNRAS, 487, 2242

Hoeijmakers, H. J., Ehrenreich, D., Kitzmann, D., et al. 2019, A\&A, 627, A165

Hu, R., Seager, S., \& Bains, W. 2012, ApJ, 761, 166

Huebner, W., \& Mukherjee, J. 2015, Planet. Space Sci., 106, 11

Huebner, W. F., \& Carpenter, C. W. 1979, Solar photo rate coefficients, Tech. rep.

Huebner, W. F., Keady, J. J., \& Lyon, S. P. 1992, Ap\&SS, 195, 1

Husser, T. O., Wende-von Berg, S., Dreizler, S., et al. 2013, A\&A, 553, A6

John, T. L. 1968, MNRAS, 138, 137

John, T. L. 1988, A\&A, 193, 189

Johnson, J. A., Gazak, J. Z., Apps, K., et al. 2012, AJ, 143, 111

Johnstone, C. P., Güdel, M., Brott, I., \& Lüftinger, T. 2015a, A\&A, 577, A28

Johnstone, C. P., Güdel, M., Lüftinger, T., Toth, G., \& Brott, I. 2015b, A\&A, 577, A27

Johnstone, C. P., Khodachenko, M. L., Lüftinger, T., et al. 2019, A\&A, 624, L10

Kislyakova, K. G., Noack, L., Johnstone, C. P., et al. 2017, Nat. Astron., 1, 878
Kitzmann, D., Heng, K., Rimmer, P. B., et al. 2018, ApJ, 863, 183 Kopparapu, R. k., Kasting, J. F., \& Zahnle, K. J. 2012, ApJ, 745, 77 Koskinen, T. T., Aylward, A. D., Smith, C. G. A., \& Miller, S. 2007, ApJ, 661, 515

Leitzinger, M., Odert, P., Greimel, R., et al. 2014, MNRAS, 443, 898

Liang, M.-C., Parkinson, C. D., Lee, A. Y. T., Yung, Y. L., \& Seager, S. 2003, ApJ, 596, L247

Line, M. R., Liang, M. C., \& Yung, Y. L. 2010, ApJ, 717, 496

Line, M. R., Vasisht, G., Chen, P., Angerhausen, D., \& Yung, Y. L. 2011, ApJ, 738,32

Lodders, K., Palme, H., \& Gail, H. P. 2009, Solar System (Berlin: Springer), 4B, 712

Loyd, R. O. P., France, K., Youngblood, A., et al. 2016, ApJ, 824, 102

Madhusudhan, N., Agúndez, M., Moses, J. I., \& Hu, Y. 2016, Space Sci. Rev., 205,285

Malik, M., Grosheintz, L., Mendonça, J. M., et al. 2017, AJ, 153, 56

Malik, M., Kitzmann, D., Mendonça, J. M., et al. 2019, AJ, 157, 170

Mendonça, J. M., Tsai, S.-m., Malik, M., Grimm, S. L., \& Heng, K. 2018, ApJ, 869,107

Miguel, Y., \& Kaltenegger, L. 2014, ApJ, 780, 166

Miller-Ricci Kempton, E., Zahnle, K., \& Fortney, J. J. 2012, ApJ, 745, 3

Molaverdikhani, K., Henning, T., \& Mollière, P. 2019a, ApJ, 883, 194

Molaverdikhani, K., Henning, T., \& Mollière, P. 2019b, ApJ, 873, 32

Molaverdikhani, K., Helling, C., Lew, B. W. P., et al. 2020, A\&A, 635, A31

Mordasini, C., Alibert, Y., Benz, W., Klahr, H., \& Henning, T. 2012, A\&A, 541, A97

Moses, J. I. 2014, Phil. Trans. R. Soc. London, Ser. A, 372, 20130073

Moses, J. I., Bézard, B., Lellouch, E., et al. 2000, Icarus, 143, 244

Moses, J. I., Visscher, C., Fortney, J. J., et al. 2011, ApJ, 737, 15

Noyes, R. W., Hartmann, L. W., Baliunas, S. L., Duncan, D. K., \& Vaughan, A. H. 1984, ApJ, 279, 763

Obermeier, C., Koppenhoefer, J., Saglia, R. P., et al. 2016, A\&A, 587, A49

Parmentier, V., \& Guillot, T. 2014, A\&A, 562, A133

Parmentier, V., Guillot, T., Fortney, J. J., \& Marley, M. S. 2015, A\&A, 574, A35

Pizzolato, N., Maggio, A., Micela, G., Sciortino, S., \& Ventura, P. 2003, A\&A, 397, 147

Poling, B., Prausnitz, J., \& Connell, J. 2000, The Properties of Gases and Liquids, Chemical Engineering Series (New York: Mcgraw-hill)

Poppenhaeger, K., Robrade, J., \& Schmitt, J. H. M. M. 2010, A\&A, 515, A98

Reiners, A., \& Mohanty, S. 2012, ApJ, 746, 43

Reiners, A., Schüssler, M., \& Passegger, V. M. 2014, ApJ, 794, 144

Ribas, I., Guinan, E. F., Güdel, M., \& Audard, M. 2005, ApJ, 622, 680

Rothman, L. S., Gordon, I. E., Barber, R. J., et al. 2010, J. Quant. Spectr. Rad. Transf., 111, 2139

Schrijver, C. J. 1995, A\&ARv, 6, 181

Schrijver, C. J., Dobson, A. K., \& Radick, R. R. 1989, ApJ, 341, 1035

Segura, A., Krelove, K., Kasting, J. F., et al. 2003, Astrobiology, 3, 689

Shulyak, D., Tsymbal, V., Ryabchikova, T., Stütz, C., \& Weiss, W. W. 2004, A\&A, 428, 993

Shulyak, D., Reiners, A., Engeln, A., et al. 2017, Nat. Astron., 1, 0184

Sing, D. K., Fortney, J. J., Nikolov, N., et al. 2016, Nature, 529, 59

Soderblom, D. R., Stauffer, J. R., MacGregor, K. B., \& Jones, B. F. 1993, ApJ, 409,624

Stock, J. W., Kitzmann, D., Patzer, A. B. C., \& Sedlmayr, E. 2018, MNRAS, 479, 865

Sudarsky, D., Burrows, A., \& Pinto, P. 2000, ApJ, 538, 885

Tennyson, J., \& Yurchenko, S. N. 2012, MNRAS, 425, 21

Tinetti, G., Drossart, P., Eccleston, P., et al. 2018, Exp. Astron., 46, 135

Tsai, S.-M., Lyons, J. R., Grosheintz, L., et al. 2017, ApJS, 228, 20

Tu, L., Johnstone, C. P., Güdel, M., \& Lammer, H. 2015, A\&A, 577, L3

Venot, O., Hébrard, E., Agúndez, M., et al. 2012, A\&A, 546, A43

Venot, O., Hébrard, E., Agúndez, M., Decin, L., \& Bounaceur, R. 2015, A\&A, 577, A33

Vernazza, J. E., Avrett, E. H., \& Loeser, R. 1981, ApJS, 45, 635

Vidotto, A. A., Fares, R., Jardine, M., Moutou, C., \& Donati, J. F. 2015, MNRAS, 449,4117

Wakeford, H. R., \& Sing, D. K. 2015, A\&A, 573, A122

Waldmann, I. P., Rocchetto, M., Tinetti, G., et al. 2015a, ApJ, 813, 13

Waldmann, I. P., Tinetti, G., Rocchetto, M., et al. 2015b, ApJ, 802, 107

Yan, F., \& Henning, T. 2018, Nat. Astron., 2, 714

Yelle, R. V. 2004, Icarus, 170, 167

Youngblood, A., France, K., Loyd, R. O. P., et al. 2016, ApJ, 824, 101

Zahnle, K., Marley, M. S., Freedman, R. S., Lodders, K., \& Fortney, J. J. 2009, ApJ, 701, L20 